\title{
Epigenetic modulator inhibition overcomes temozolomide chemoresistance and antagonizes tumor recurrence of glioblastoma
}

\author{
Byoung-San Moon, ${ }^{1,2,3,4}$ Mingyang Cai, ${ }^{2}$ Grace Lee, ${ }^{2}$ Tong Zhao, ${ }^{5}$ Xiaofeng Song, ${ }^{6}$ Steven L. Giannotta, ${ }^{1}$ Frank J. Attenello, ${ }^{1}$ \\ Min Yu, ${ }^{2}$ and Wange Lu ${ }^{2,5}$ \\ 'Department of Neurosurgery and 'Broad Center for Regenerative Medicine and Stem Cell Research, Keck Department of Stem Cell Biology and Regenerative Medicine, Keck School of Medicine, University \\ of Southern California, Los Angeles, California, USA. ${ }^{3}$ Therapeutics and Biotechnology Division, Drug Discovery Platform Research Center, Korea Research Institute of Chemical Technology (KRICT), Daejeon, \\ Korea. ${ }^{4}$ Department of Biotechnology, Chonnam National University, Yeosu, Korea. ${ }^{5}$ State Key Laboratory of Medicinal Chemical Biology and College of Life Sciences, Nankai University, Tianjin, China. \\ ${ }^{6}$ Department of Biomedical Engineering, Nanjing University of Aeronautics and Astronautics, Nanjing, China.
}

\begin{abstract}
Clioblastoma multiforme (GBM) heterogeneity causes a greater number of deaths than any other brain tumor, despite the availability of alkylating chemotherapy. GBM stem-like cells (CSCs) contribute to GBM complexity and chemoresistance, but it remains challenging to identify and target GSCs or factors that control their activity. Here, we identified a specific CSC subset and show that activity of these cells is positively regulated by stabilization of methyl CpC binding domain 3 (MBD3) protein. MBD3 binds to CK1A and to BTRCP E3 ubiquitin ligase, triggering MBD3 degradation, suggesting that modulating this circuit could antagonize CBM recurrence. Accordingly, xenograft mice treated with the CK1A activator pyrvinium pamoate (Pyr-Pam) showed enhanced MBD3 degradation in cells expressing high levels of $\mathrm{O}^{6}$-methylguanine-DNA methyltransferase (MCMT) and in CSCs, overcoming temozolomide chemoresistance. Pyr-Pam blocked recruitment of $\mathrm{MBD} 3$ and the repressive nucleosome remodeling and deacetylase (NuRD) complex to neurogenesisassociated gene loci and increased acetyl-histone $\mathrm{H3}$ activity and CSC differentiation. We conclude that CK1A/BTRCP/ MBD3/NuRD signaling modulates CSC activation and malignancy, and that targeting this signaling could suppress CSC proliferation and CBM recurrence.
\end{abstract}

\section{Introduction}

Glioblastoma multiforme (GBM; grade IV glioma) is a heterogeneous and highly aggressive primary brain tumor with a 14.6-month survival, even after surgery, chemotherapy, and radiation therapy (1). Treatments are limited by the high degree of GBM heterogeneity and the presence of GBM stem-like cells (GSCs), which exhibit self-renewal and differentiation activities resembling those of neural progenitor cells (NPCs) (2-4). GSCs are also implicated in tumor initiation, progression, relapse, and metastasis $(2,4)$. Development of effective treatment therefore requires identification of markers targeting GSCs as well as an understanding of mechanisms underlying GSC activity.

Studies suggest that GSCs originate from NPCs in the central nervous system, and that molecular pathways governing selfrenewal and multilineage differentiation of GSCs parallel those in NPCs (5-7). Although prior studies have investigated processes common to both NPC cell fate and GBMs (8-10), it remains unclear whether epigenetic mechanisms known to regulate NPC activity (11) also affect GBMs and could be targeted as treatment.

Conflict of interest: The authors have declared that no conflict of interest exists. Copyright: (c) 2020, American Society for Clinical Investigation.

Submitted: February 2, 2019; Accepted: July 16, 2020; Published: October 5, 2020

Reference information: J Clin Invest. 2020;130(11):5782-5799.

https://doi.org/10.1172/JCI127916.
Methyl CpG binding domain protein 3 (MBD3), a core component of the repressive nucleosome remodeling and deacetylase (NuRD) complex, plays a critical role in stem cell pluripotency, differentiation, and cell death (12-14). So far, 5 human MBD proteins have been identified and characterized (MBD1, MBD2, MBD3, MBD4, and MECP2) (15-18). Unlike other family members, which recognize $5^{\prime}$-methyl-cytosine-modified (5'-mC-modified) DNA, MBD3 specifically recognizes $5^{\prime}$-hydroxymethyl-cytosine $\left(5^{\prime}\right.$ $\mathrm{hmC}$ ), an epigenetic marker highly enriched in stem cells and cancer cells $(15,19-23)$. MBD3 specifically functions in maintenance of transcriptionally repressed chromatin $(20,24)$. In human cancers, loss- or gain-of-function mutations in $M B D 3$ reportedly dysregulate gene expression in a manner that promotes tumorigenesis (25-27). Despite emerging evidence that MBD3 plays critical roles in both stem cells and cancer, it remains unknown whether MBD3 plays a role in cancer stem cells.

Here, we show that MBD3 destabilization overcomes temozolomide (TMZ) chemoresistance by promoting neural differentiation of the GSC subpopulation, a process regulated by CK1A/ BTRCP/NuRD signaling. Using RNA sequencing, tissue microarrays (TMAs), sphere-formation assays, and xenograft models, we first show that MBD3 degradation promotes differentiation of $\mathrm{CD} 44^{+} \mathrm{CD} 133^{+} \mathrm{CXCR} 4^{+}$triple-positive GSCs and inhibits their proliferation in vitro and in vivo. Using mass spectrometry (MS) and Western blot analysis, we then identified the E3 ligase $\beta$-trans- 
ducin repeats-containing protein (BTRCP), which serves as the substrate recognition subunit for $\mathrm{SCF}^{\mathrm{BTRCP}} \mathrm{E} 3$ ubiquitin ligases (28), and casein kinase $1 \alpha(\mathrm{CK} 1 \mathrm{~A})$, as MBD3 interaction partners. MBD3 protein was serine phosphorylated by CK1A at sites recognized by $\mathrm{BTRCP}$, leading to $\mathrm{MBD} 3$ ubiquitination and proteasomal degradation. In addition, the CK1A activator pyrvinium pamoate (Pyr-Pam), an FDA-approved oral anthelmintic drug, promoted MBD3 protein degradation and prevented accumulation of the MBD3-NuRD complex on target gene loci functioning in GSC differentiation. In vitro and in vivo analyses performed in GBM lines or a patient-derived xenograft (PDX) model confirmed that suppression of GBM propagation and resistance after TMZ treatment is controlled by MBD3 destabilization and, importantly, is dependent on CK1A activation. Collectively, our results reveal that MBD3 is a potentially new drug target in a specific GSC subpopulation and that $\mathrm{CK} 1 \mathrm{~A} / \mathrm{BTRCP} / \mathrm{MBD} 3 / \mathrm{NuRD}$ signaling is a mechanism underlying GSC differentiation.

\section{Results}

MBD3 promotes expression of $\mathrm{CD} 44^{+} \mathrm{CD} 133^{+} \mathrm{CXCR} 4^{+}$triple-positive GSC markers. The cell surface protein CD133 (also known as prominin-1) reportedly marks GBM cells with stem-like properties and has been used to enrich those populations $(2,29,30)$. However, CD133 marks a large percentage of tumor cells and reportedly lacks specificity as a GSC marker $(29,31)$. Thus, we searched for novel GSC markers by identifying genes differentially expressed in patient GBM $(n=20)$ versus normal human brain specimens ( $n$ = 19) in our previous study (Figure 1A, Supplemental Table 1, Supplemental Dataset 1, and ref. 32; supplemental material available online with this article; https://oi.org/10.1172/JCI127916DS1). Among the 5075 genes upregulated in GBM patient specimens, we selected 69 known markers of either stem cells or tumorinitiating cells in various cancers or NPCs (Supplemental Figure 1, A and B, and Supplemental Table 2). We then ranked each using a fold-change cutoff of 10 and $P \leq 1 \times 10^{-2}$ and analyzed the top 20 by immunohistochemistry using GBM tissue supported by the Human Protein Atlas (http://www.proteinatlas.org/) or stained tissue from a TMA (Supplemental Figure 2, A and B). We chose 3 candidates, CD44, CD133, and CXCR4, based on quantification of fluorescence intensity of immunostained TMA samples (Figure $1 \mathrm{~B}$ and Supplemental Figure 2C). Compared with the remaining 17 markers, CD44, CD133, and CXCR4 expression was much higher in GBM tissues from high-grade (stages III and IV) tumors than in normal brain tissues or low-grade (stages II and II/III) tumors (Figure 1B and Figure 2, $\mathrm{A}^{-} \mathrm{C}$ ). We then carried out sphereformation assays to determine whether $\mathrm{CD} 44^{+} \mathrm{CD} 133^{+} \mathrm{CXCR} 4^{+}$triple-positive GBM cells formed spheres more efficiently than did single-positive, double-positive, or triple-negative populations. Indeed, $\mathrm{CD} 44^{+} \mathrm{CD} 133^{+} \mathrm{CXCR} 4^{+}$triple-positive spheres exhibited significantly wider diameters than did single, double-positive, or triple-negative spheres after 5 days of culture in vitro (Supplemental Figure 3, A and B). Moreover, after injecting corresponding subpopulations into immunocompromised mice, the tumor-forming capacity of triple-positive cells was significantly higher than that of single-positive or unsorted cells, both of which formed tumors at low frequency and only following injection of a large number of cells $\left(5 \times 10^{3}\right)$ (Figure 1C and Supplemental Figure 3C). Relevant to self-renewal, $\mathrm{CD} 44^{+} \mathrm{CD} 133^{+} \mathrm{CXCR} 4^{+}$triple-positive single cells or groups of cells showed greater secondary sphere-forming ability than did triple-negative cells (Figure 1, D and E, and Supplemental Figure 3D). These observations suggest overall that coexpression of CD44, CD133, and CXCR4 marks a subset of GSCs.

Numerous studies suggest that GBM tumor-initiating cells originate from cells in the subventricular zone (SVZ), including NPCs $(5-7,33)$, and we recently identified MBD3 protein as an epigenetic determinant of SVZ NPC fate (13). Interestingly, MBD3 expression increases in parallel with GBM grade (Supplemental Figure 3, E and F), and its expression significantly correlates with expression of the GSC markers identified above (MBD3 and CD44, $R=0.8556$; MBD3 and CD133, $R=0.9464$; MBD3 and CXCR4, $R$ $=0.9227$ ) (Supplemental Figure $3 G$ ). Moreover, low expression of both MBD3 and those GSC markers correlates with better survival in The Cancer Genome Atlas (TCGA) database (https://www. proteinatlas.org/ENSG00000071655-MBD3/pathology/tissue/ glioma) (Supplemental Figure 3H and Supplemental Table 3). After assessing CD44, CD133, and CXCR4 expression in $\mathrm{MBD}^{\text {hi }}$ or $\mathrm{MBD}^{\text {lo }}$ fractions of GBM cells, we discovered that expression of all 3 was higher in the $M B D 3^{\text {hi }}$ fraction (Figure $1, F$ and $G$ ). In particular, cells from the MBD3 ${ }^{\text {hi }}$ fraction showed higher secondary sphereforming ability than those from the $\mathrm{MBD}^{\text {lo }}$ fraction (Figure $1, \mathrm{H}$ and I, and Supplemental Figure 3I). To determine whether MBD3 activity alters GSC marker expression, we transfected the human GBM cell line T98G with MBD3 or control vectors and undertook RT-qPCR analysis. MBD3-overexpressing cells showed significantly increased levels of CD44, CD133, and CXCR4; OCT4, NANOG, SOX2, and KLF4 (pluripotency markers), and PAX6 and NESTIN (self-renewal markers) (Supplemental Figure 3J), results confirmed by immunoblot analysis (Supplemental Figure 3K). Moreover, to evaluate MBD3 function in GSC self-renewal, we transduced USC02 human primary GSCs with $M B D 3$ or shMBD3 lentiviral vectors and measured sphere-forming capacity. MBD3-knockdown cells showed significantly decreased sphere-forming activity, while MBD3-overexpressing cells showed no change in sphere size or number relative to controls (Supplemental Figure 3, L and $\mathrm{M})$. These data suggest that $\mathrm{CD} 44^{+} \mathrm{CD} 133^{+} \mathrm{CXCR} 4^{+}$triple-positive fractions represent potentially novel subpopulations showing GSC potential, and that MBD3 promotes expression of these markers.

Increased MBD3 stability correlates with maintenance of GSC stemness. MBD3 protein destabilization promotes neurogenesis during mouse cortical development (13). To evaluate whether MBD3 stability is associated with GBM progression or GSC activity, we assessed MBD3 stability in T98G GSCs. We first selected $\mathrm{CD} 133^{+} \mathrm{CD} 44^{+} \mathrm{CXCR} 4^{+}$triple-positive populations of $\mathrm{T} 98 \mathrm{G}$ cells and plated them as adherent cells. We then withdrew basic fibroblast growth factor (bFGF) to induce differentiation. Following induction, we observed that MBD3 protein levels gradually decreased (Figure 2A). Moreover, high CD44, CD133, and CXCR4 expression seen before induction in T98G GSCs also significantly decreased (Figure 2A), suggesting that GSC differentiation positively correlated with decreased MBD3 protein level. We also observed that GSCs were multipotent and differentiated into both neurons and glial cells (Figure 2, A and B).

In NPCs, MBD3 is regulated at the protein rather than the mRNA level (13). Thus, we analyzed MBD3 protein stability in 
A

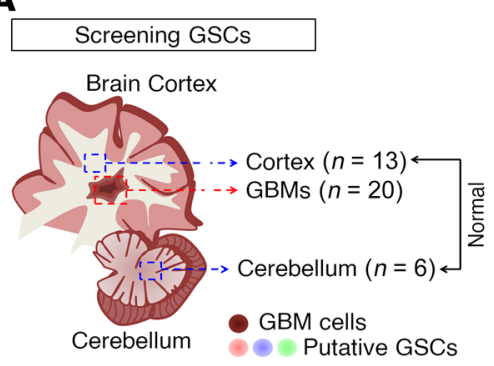

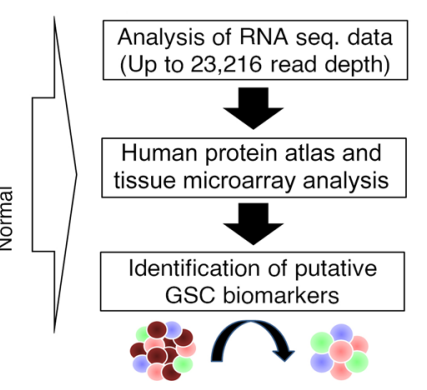

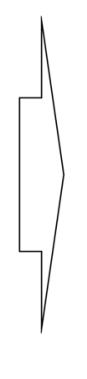

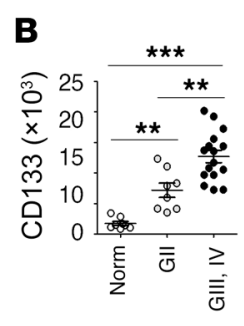

C

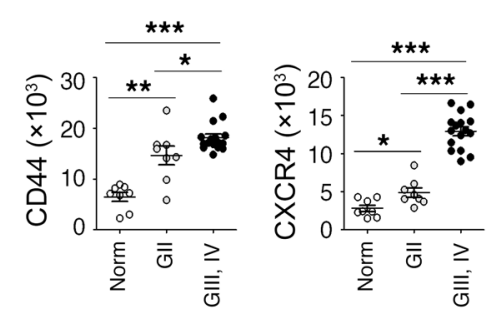

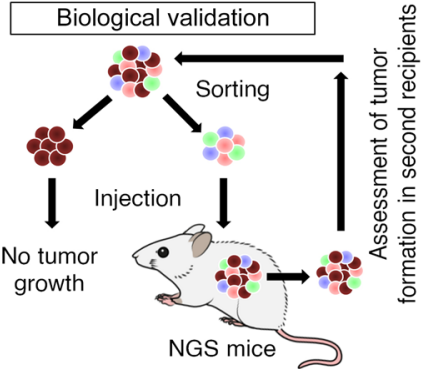

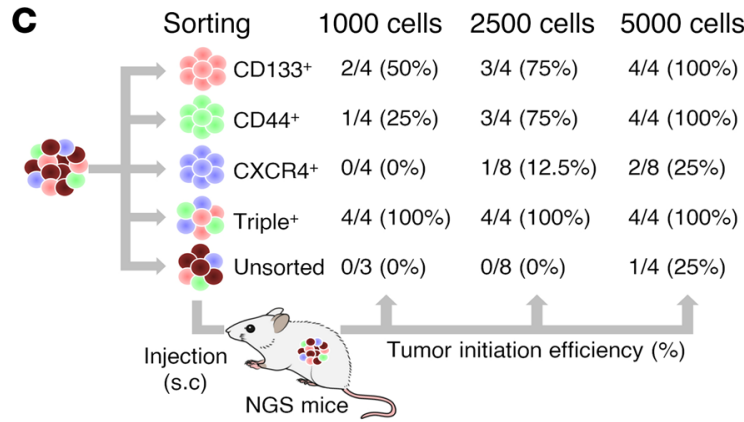

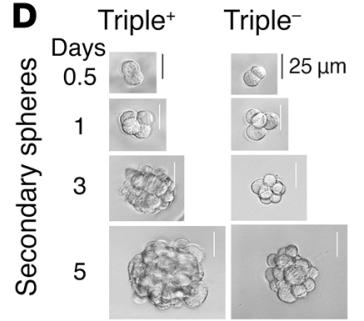

$\mathbf{E}$
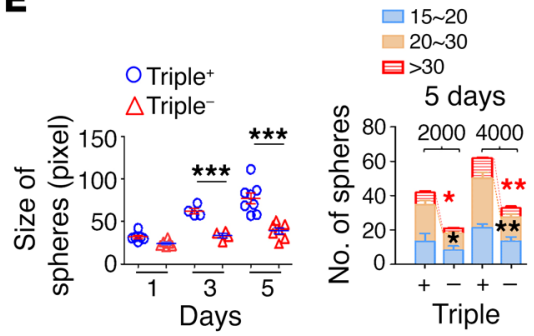

$\mathbf{F}$
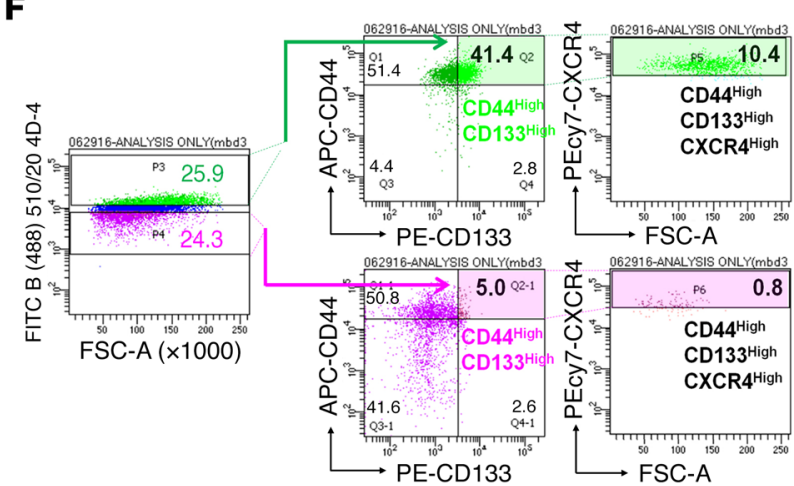
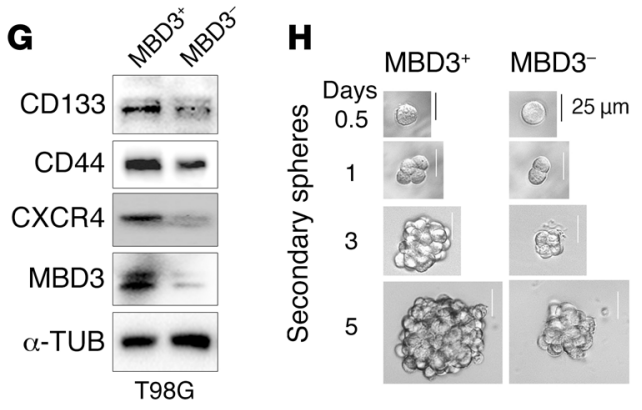

I

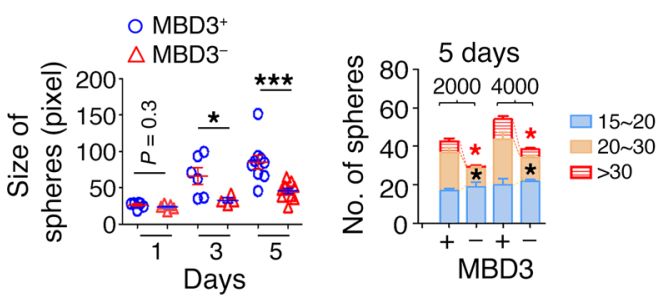

Figure 1. A CD44+CD133+CXCR4+ triple-positive GBM subpopulation exhibits previously uncharacterized GSC properties, and MBD3 regulates their marker expression and stemness. (A) Schematic overview for identification of putative glioblastoma stem cell (CSC) populations. (B) TMA analysis (normal brain, $n=8$; grade II [GII], $n=8$; GIII, IV, $n=16$ ). (C) The number of tumors for each condition is presented. (D) Relative sizes of secondary spheres. (E) Relative sizes and numbers of spheres in Supplemental Figure 3D. Numbers in the color key above the right panel indicate the ranges of sphere sizes (pixels) of CD133+CD44+CXCR4+ triple-positive (+) and -negative (-) populations; 2000 and 4000 refer to the number of cells used. (F) FACS analyses of the

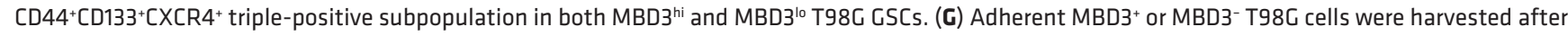
culture for 2 days in N2 medium with bFGF, and lysates were immunoblotted with indicated antibodies. (H) FACS-isolated MBD3 ${ }^{+}$or MBD3- cells were seeded as single cells in ultra-low-attachment 96 -well plates for 1,3 , or 5 days to allow sphere formation. Relative sizes of spheres shown in left panel. (I) Relative sizes and numbers of spheres shown in Supplemental Figure 31 . Numbers in the color key next to the right panel indicate the ranges of sphere sizes (pixels) of MBD3-positive (+) and -negative (-) populations; 2000 and 4000 refer to the number of cells used. In B, D, E, and G-I, quantification was performed using ImageJ software (NIH). GBM, glioblastoma multiforme. Data are presented as the mean \pm SD and are representative of at least 3 independent experiments. Statistical significance was tested with 1-way ANOVA with Tukey's multiple-comparison test (B, D, and H) or unpaired, 2-tailed Student's $t$ test $(\mathbf{E}$ and $\mathrm{I}) .{ }^{*} P<0.05 ;{ }^{* *} P<0.005 ;{ }^{* *} P<0.0005$. 
A

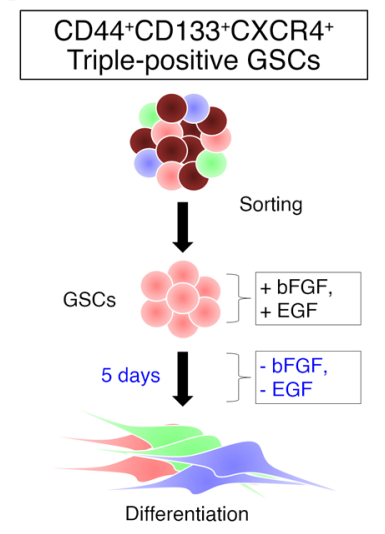

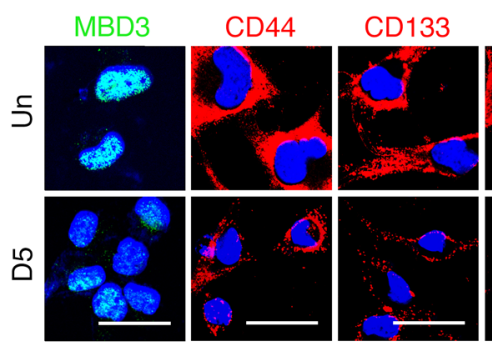
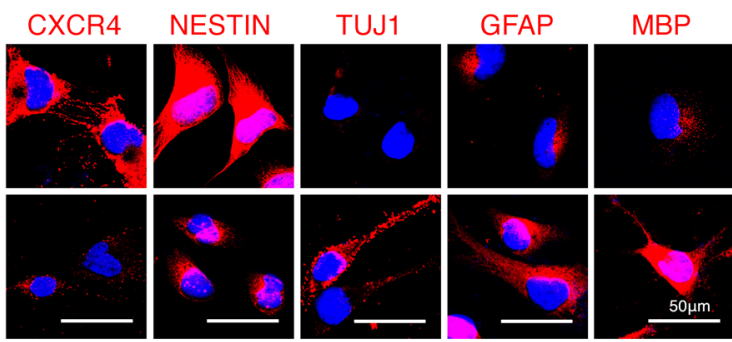

B
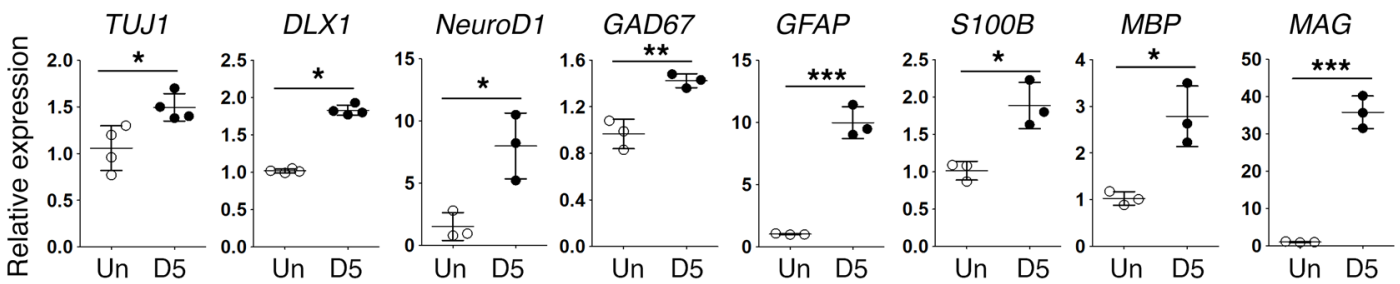

C
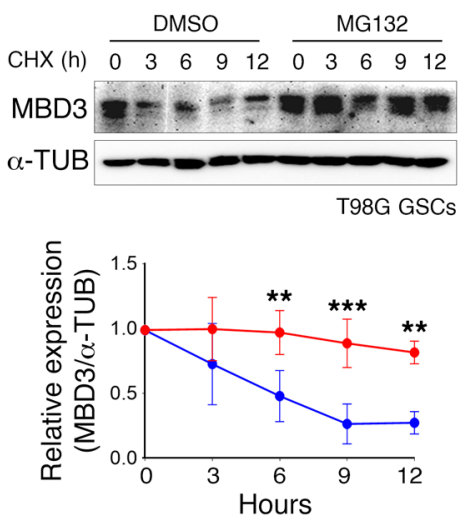

D
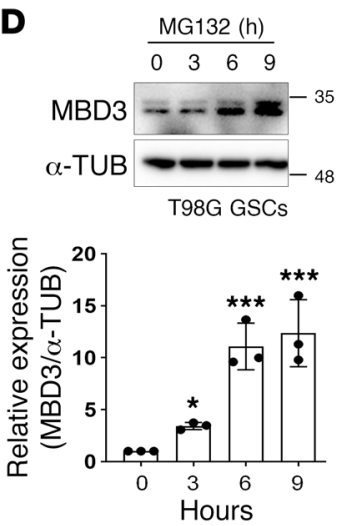

$\mathbf{E}$

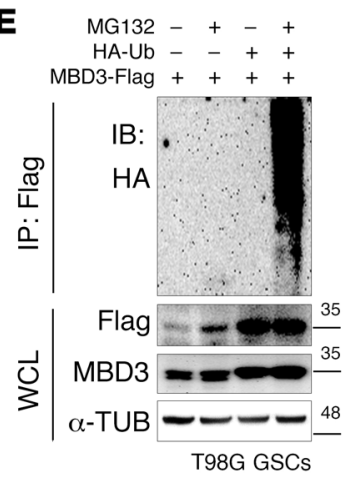

$F$

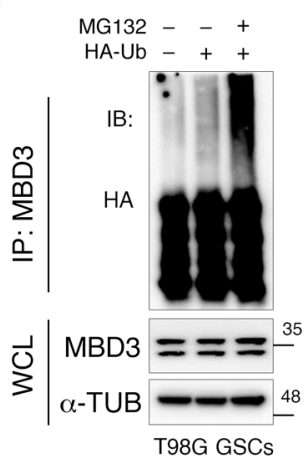

Figure 2. Degradation and ubiquitination of MBD3 in GSCs. (A) Left: CD44+CD133+CXCR4+ triple-positive cells sorted by FACS from T98G GSCs at a purity of $99.8 \%$ were grown on coverslips to monitor differentiation capacity over 5 days. Right: Undifferentiated cells (Un) and cells after 5 days of differentiation (D5) were analyzed by immunofluorescence with the indicated antibodies. Nuclei were counterstained with DAPI. Scale bars: $50 \mu \mathrm{m}$. (B) qPCR analysis of the indicated mRNAs in samples from $\mathbf{A}(n=3$ or 4). (C) Immunoblot (IB) analysis of MBD3 and $\alpha$-tubulin in lysates of T98C GSCs, treated with cycloheximide (CHX) for indicated times (h, hours) and with or without MG132. (D) T98G GSCs were treated with MG132 for 0, 3, 6, and 9 hours before harvesting. Whole-cell lysates (WCLs) were immunoblotted using the indicated antibodies. (E and $\mathbf{F}$ ) Ubiquitination assay of overexpressed or endogenous MBD3 using indicated lysates of cells transfected with MBD3-Flag and HA-Ub expression vectors and treated 1 day later with MG132 for 6 hours before harvesting. After lysis, immunoprecipitation (IP) was performed with Flag-M2 beads or MBD3 antibody. WCLs were analyzed by IB with the indicated antibodies. Data are presented as the mean $\pm S D$. ${ }^{*} P<0.05 ;{ }^{* *} P<0.005 ;{ }^{* *} P<0.0005$ by unpaired, 2 -tailed Student's $t$ test.

GBM cells. Following inhibition of protein synthesis with cycloheximide (CHX), the MBD3 half-life in T98G GSCs was approximately 6 hours, a period significantly extended in cells treated with the proteasomal inhibitor MG132 (Figure 2C). Endogenous MBD3 protein levels in T98G GSCs increased time dependently following MG132 treatment (Figure 2D). Both overexpressed and endogenous MBD3 proteins were polyubiquitinated and accumulated in MG132-treated T98G GSCs (Figure 2, E and F). These findings suggest that factors that regulate MBD3 protein stability in GSCs and normal NPCs are comparable.

The E3 ligase BTRCP regulates $M B D 3$ protein stability in $G B M$ cells/GSCs. To define mechanisms governing MBD3 protein stabil- ity in GBM, we assessed MBD3 protein interactomes by performing mass spectrometry (MS) analysis of Flag-MBD3 overexpressed in T98G cells. That analysis identified SKP1, CUL1/2/3/5, and BTRCP complex as putative binding partners (Figure 3A and Supplemental Table 4). Further inspection revealed that amino acids 36 to 110 in the MBD3 N-terminus contained ${ }_{38} \mathrm{PSGX}_{(n)} \mathrm{S}_{45}$ and ${ }_{84} \mathrm{DSSX}_{(n)} \mathrm{S}_{106}$ sequences, which match the degron motif (DSGXXS) recognized by BTRCP when both serines are phosphorylated (Figure 3B). Coimmunoprecipitation (co-IP) assays in HEK293T cells transfected with MBD3 and/or BTRCP expression constructs confirmed an MBD3-BTRCP interaction (Figure 3, C and D). By contrast, the MBD3 $(\triangle 2 \mathrm{D})$ mutant, which lacks both degron motifs, 
A

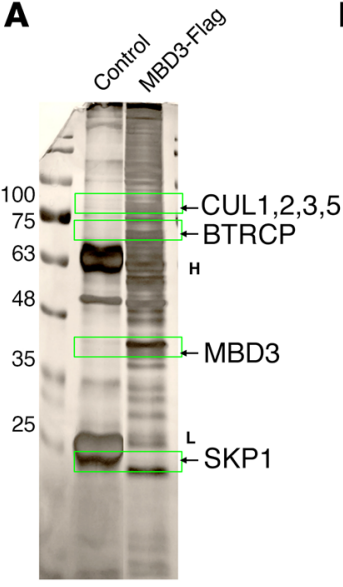

B

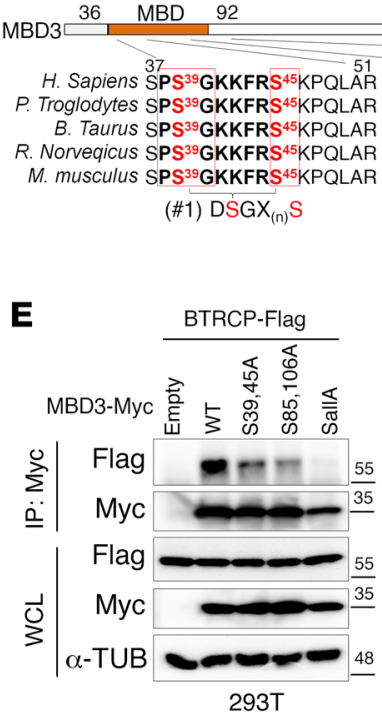

$293 T$

$\mathrm{CC}$

1110

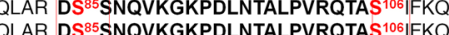
P. TS R. Norveqicus SPS ${ }^{39}$ GKKFRS $^{45}$ KPQLAR DS ${ }^{85}$ SNQVKGKPDLNTALPVRQTAS ${ }^{106} \mid \mathrm{FKQ}$ M. musculus SPS ${ }^{39} \mathrm{GKKFRS}^{45} \mathrm{KPQLAR}$ DS ${ }^{85}$ SNQVKGKPDLNTALPVRQTAS ${ }^{106} \mid \mathrm{FKQ}$ $\begin{array}{ll}\text { (\#1) DSGX } & \\ & \end{array}$

$\mathbf{F}$

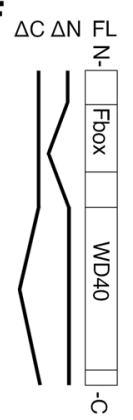

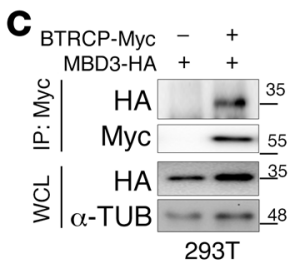

D $_{\text {BTRCP-Myc }++}+$ MBD3-Flag - +

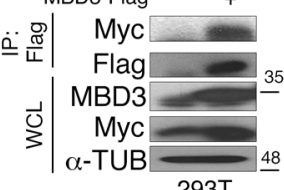

293T

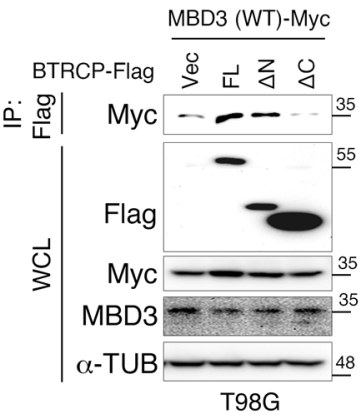

G

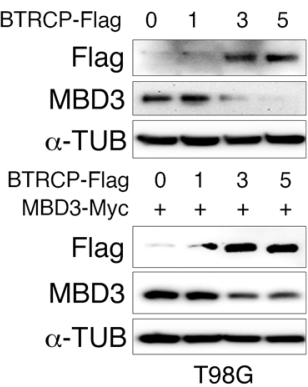

H

MG132 - + - + - + $\mathrm{HA}-\mathrm{Ub}--+\overline{+}+$ MBD3-Myc ++++++
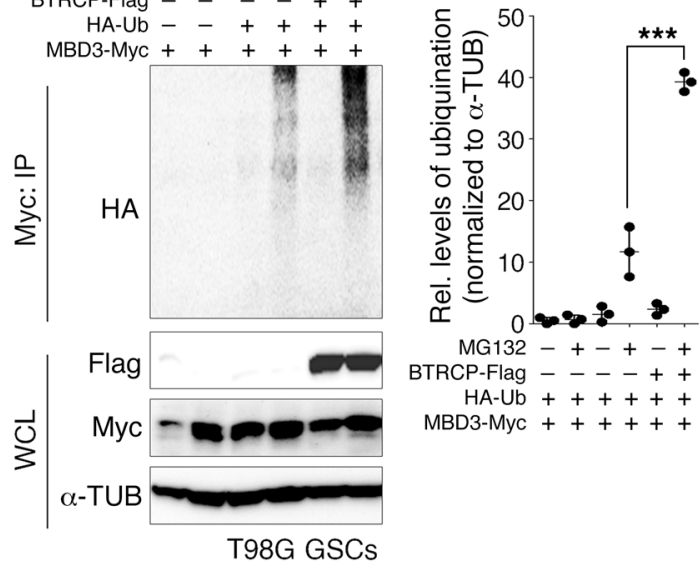

$\mathrm{HA}-\mathrm{Ub}++++++$ MBD3-Myc ++++++
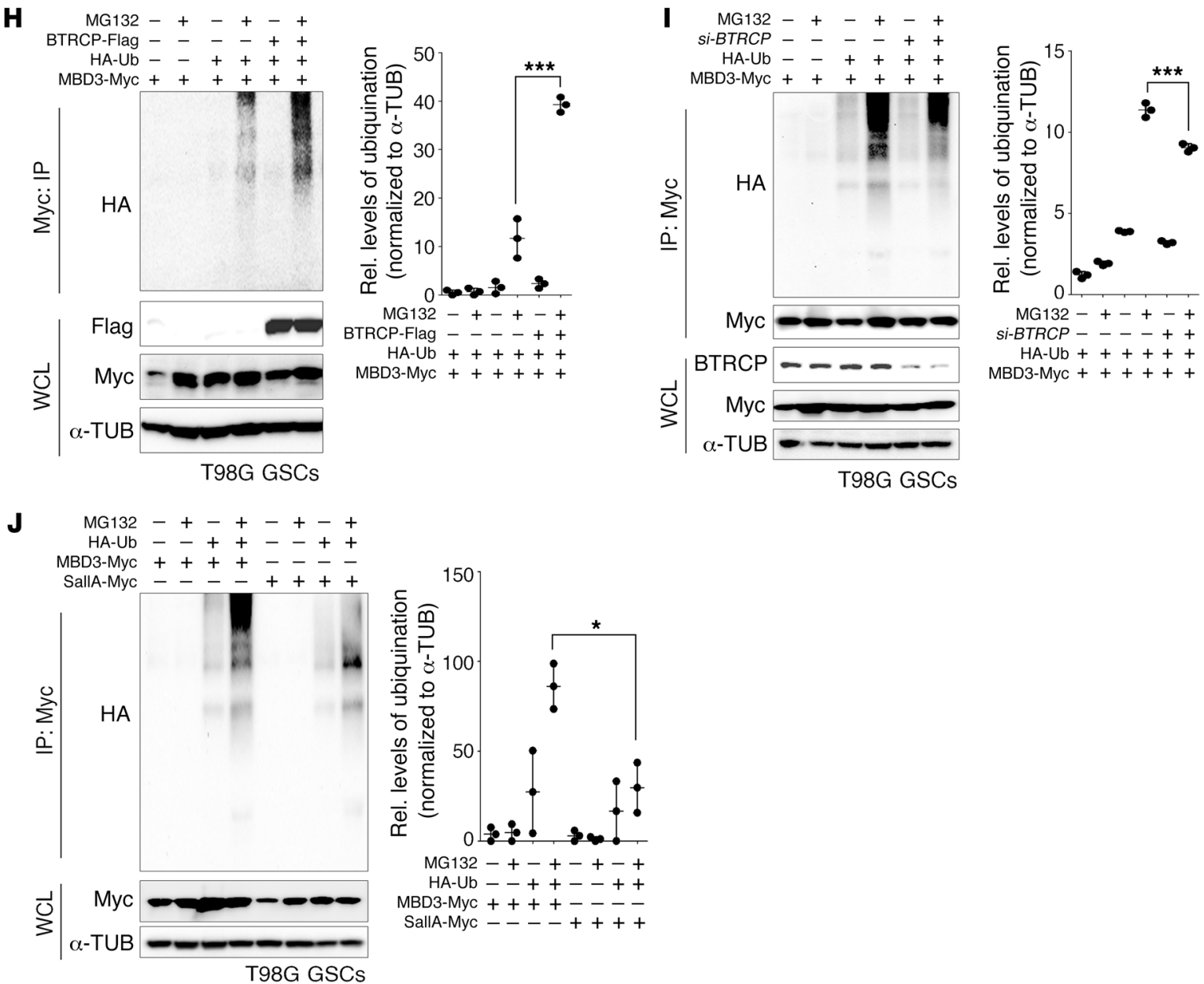

MG132 - + - + - + + $\mathrm{HA}-\mathrm{Ub}--++-++$ Sall-Myc ++++---T98G GSCs SallA-Myc ---++++ 
Figure 3. BTRCP is an E3 ubiquitin ligase promoting MBD3 polyubiquitination and subsequent proteasomal degradation. (A) Anti-Flag IPs from T98G-Flag-MBD3 cells were resolved by SDS-PAGE and visualized by silver staining. Specific bands were excised and analyzed using mass spectrometry. $\mathrm{H}$, IgG heavy chain; L, IgG light chain. (B) Diagram of conserved domains of MBD3 protein (upper) and the 2 degron $\left(\operatorname{DSCX}_{(n)} \mathrm{S}\right)$ motifs of MBD3 orthologs (lower). MBD, methyl CpG binding domain. (C and D) IP with anti-Myc or Flag-M2 beads of respective lysates made from cells transfected with either HA-MBD3 with or without Myc-BTRCP or Myc-BTRCP plus control vector or Flag-MBD3. (E) IP using Myc-conjugated beads in lysates from cells transfected with BTRCP-Flag plus control vector or WT or SA double (S39A/S45A or S85A/S106A) or quadruple (SallA) mutant MBD3-Myc. Whole-cell lysates (WCLs) were immunoblotted using the indicated antibodies. (F) Diagram of BTRCP deletion mutants (left) and IP using Flag-M2 beads of lysates from cells transfected with MBD3Myc plus control vector, WT BTRCP-Flag, or indicated BTRCP deletion mutants (right). WCLs were immunoblotted with the indicated antibodies. (C) T98C cells were transfected with empty vector or increasing levels of Flag-tagged BTRCP expression vector. WCLs were collected 48 hours later and immunoblotted (IB) with the indicated antibodies. ( $\mathbf{H}$ and $\mathbf{I}$ ) Effect of BTRCP overexpression or knockdown on MBD3 polyubiquitination. Sorted T98G GSCs were transfected with the indicated vectors and treated with MG132. Left: WCLs were immunoprecipitated with the indicated beads recognizing Myc. Right: Quantification of polyubiquitinated MBD3 normalized to $\alpha$-tubulin $(n=3)$. (J) Ubiquitination of overexpressed MBD3 in lysates of T98G GSCs transfected with WT or SallA mutant Myc-MBD3 plus HA-Ub expression vectors and treated 1 day later with MG132 for 6 hours before harvest. After lysis, IP was performed with Flag-M2 beads. WCLs were analyzed by IB with indicated antibodies. Data are representative of at least 3 independent experiments. BTRCP, $\beta$-transducin repeats-containing protein. Data are presented as the mean $\pm \mathrm{SD}$. ${ }^{*} P<0.05$; ${ }^{* *} P<0.0005$ by 1-way ANOVA with Tukey's multiple-comparison test.

did not bind BTRCP (Supplemental Figure 4A), suggesting that the $\mathrm{N}$-terminus containing the 2 degron motifs is required. Phosphorylation is often required for substrate binding to an E3 ligase (34). To assess whether this is a factor in BTRCP-MBD3 binding, we generated MBD3 serine-to-alanine (SA) double mutants (S39A/ S45A or S85A/S106A) or quadruple mutants (S39A/S45A/S85A/ S106A [also referred to as SallA]) and then transiently transfected HEK293T cells with a BTRCP expression vector plus one of the SA mutants or the full-length MBD3 plasmid. BTRCP's interaction with MBD3 decreased in cells cotransfected with MBD3 double mutants (S39A/S45A or S85A/S106A) and was largely abrogated following transfection with the quadruple (SallA) mutant, supporting the idea that the $\mathrm{N}$-terminal degron motifs and more than one of these serine sites are critical for BTRCP binding (Figure 3E). Misexpression of the SallA mutant promoted comparable phenotypes in T98G GSCs (Supplemental Figure 4B), suggesting serine phosphorylation of MBD3 N-terminal degron motifs is necessary for BTRCP binding. Next, to map BTRCP domains required for MBD3 interaction in T98G cells, we generated BTRCP mutants and assessed their interaction (Figure 3F). Specifically, the BTRCP C-terminus, which contains 7 WD40 repeats, recognizes the phosphorylated degron motif of substrates, promoting their degradation (34). Indeed, analysis of BTRCP deletion mutants indicated that the BTRCP C-terminus interacts with MBD3 (Figure 3F), whereas deletion of the $\mathrm{N}$-terminus did not significantly change the binding affinity for MBD3 (Figure 3F).

We next asked whether BTRCP regulates MBD3 protein abundance. BTRCP overexpression in T98G cells promoted a decrease in levels of both endogenous and exogenous MBD3 (Figure 3G), effects not seen in MG132-treated cells (Supplemental Figure 4C), suggesting that MBD3 undergoes BTRCP-dependent proteasomal degradation. Thus, we asked whether BTRCP promoted MBD3 ubiquitination. BTRCP overexpression in T98G GSCs significantly increased ubiquitination levels of MBD3 (Figure 3H), whereas BTRCP knockdown or overexpression of the SallA mutant significantly decreased MBD3 ubiquitination levels (Figure 3, I and J). Furthermore, overexpression of BTRCP lacking the WD40 domain in T98G GSCs substantially decreased ubiquitination of MBD3 (Supplemental Figure 4D). Collectively, these results indicate that BTRCP, through its WD40 domain, ubiquitinates and promotes degradation of MBD3 protein in GBM cells and in GSCs.

CK1A activation promotes MBD3 ubiquitination. Our observation that multiple serines in the MBD3 N-terminal degron motifs likely interact with BTRCP prompted us to search for protein kinase(s) responsible for MBD3 phosphorylation at those sites. Using Group-based Prediction System (GPS) software (version 3.0) (http://gps.biocuckoo.org/), we identified CK1A as a candidate (Figure 4A). To assess whether MBD3 and CK1A form a complex, we performed co-IP with Myc-MBD3 or HA-MBD3 and Flag-CK1A expressed in HEK293T cells and observed specific interaction under physiological conditions (Figure 4B). To assess potential phosphorylation of MBD3 by CK1A, we overexpressed both Myc-MBD3 and Flag-CK1A in HEK293T cells and performed Western blot analysis of Myc immunoprecipitates with an anti-phosphoserine antibody. Overexpression of WT CK1A dose-dependently increased MBD3 phosphorylation levels relative to controls (Figure 4C). Next, to determine whether CK1A-dependent phosphorylation altered MBD3 stability, we pretreated T98G cells with increasing doses of the CK1A inhibitor D4476 and observed dose-dependent stabilization of endogenous MBD3 protein (Supplemental Figure 4E). We then performed shRNA-mediated CK1A knockdown in USC02 GSCs, a mesenchymal GBM subtype, and treated cells with $\mathrm{CHX}$. MBD3 protein half-life remarkably increased in CK1A-knockdown cells relative to shScramble (Figure 4D and Supplemental Figure 4F). Moreover, prolonged MBD3 protein half-life by CK1A knockdown was also repeated in HEK293T cells or USC02 GSCs treated with the CK1A inhibitor D4476 (Figure 4D, lower, Supplemental Figure 4G, and ref. 35). Conversely, treatment of T98G GSCs with the CK1A activator Pyr-Pam significantly decreased the stability of endogenous MBD3 protein dose dependently (Figure 4E), an effect rescued by treatment with $\mathrm{D} 4776$, also dose dependently (Figure $4 \mathrm{~F}$ ). To further confirm an effect of CK1A on MBD3 stability, we transiently overexpressed either full-length CK1A or CK1A plus a corresponding dominant negative (DN) construct in T98G cells and assessed MBD3 ubiquitination. CK1A overexpression increased MBD3 polyubiquitination; however, cotransfection of CK1A with the DN form significantly abolished that effect (Figure 5A). Next, we asked whether phosphorylation by CK1A functions in BTRCPmediated MBD3 ubiquitination and degradation. BTRCP overexpression in T98G cells increased MBD3 ubiquitination, and cotransfection with CK1A significantly increased MBD3 ubiquitination dose dependently (Figure 5B). As confirmation, we treated cells with Pyr-Pam plus MG132 and determined that MBD3 destabilization by Pyr-Pam occurred via polyubiquitin-dependent 

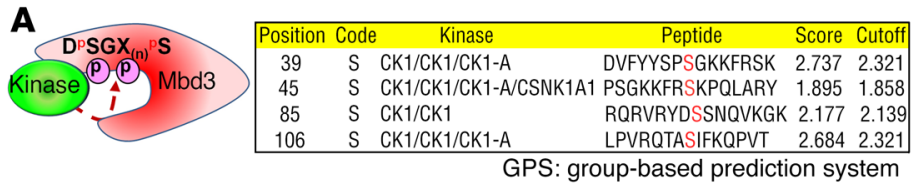

B

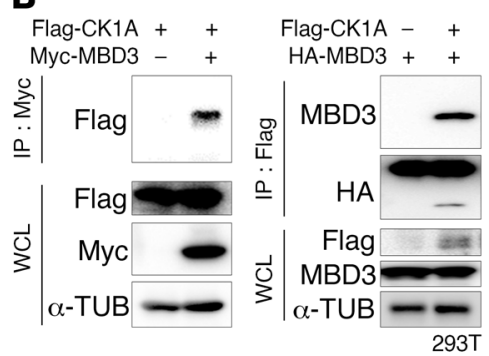

$\mathbf{E}$

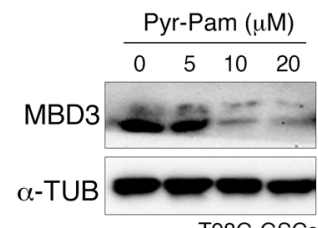

T98G GSCs
C

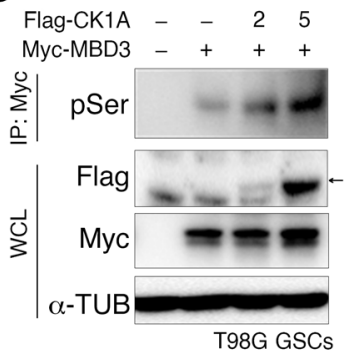

$\mathbf{F}$
D

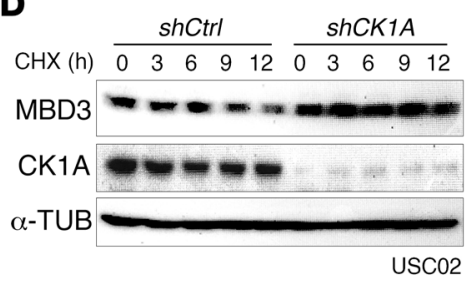

USC02

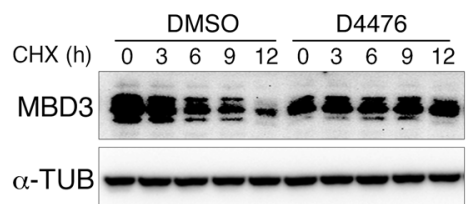

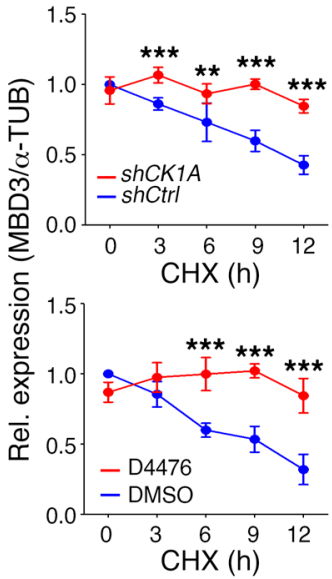
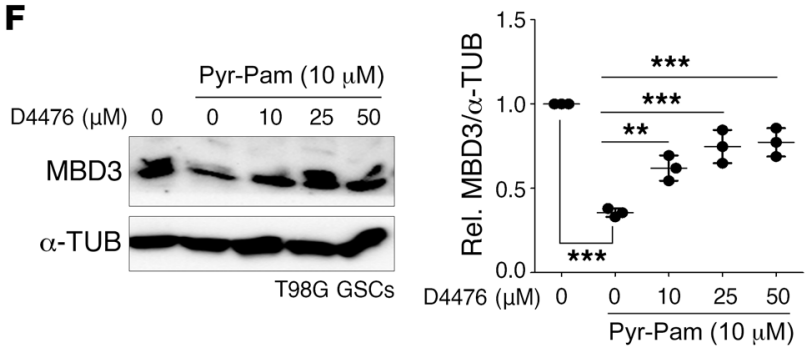

Figure 4. CK1A-mediated phosphorylation of serine residues in MBD3 degron motif promotes MBD3 degradation. (A) Group-based Prediction System software (version 2.1) predicts that CK1A phosphorylates MBD3 serines 39, 45, 85, and 106 at degron domains. (B) IP using Myc or Flag-M2 beads from respective lysates of HEK293T cells harboring Flag-tagged CK1A in the presence or absence of Myc-MBD3 or harboring HA-MBD3 plus either control vector or Flag-CK1A. Whole-cell lysates (WCLs) were immunoblotted (IB) with the indicated antibodies. (C) WCLs of T98G GSCs transfected with Myc-tagged MBD3 or control vectors plus increasing levels of Flag-tagged CK1A vector were immunoprecipitated with the indicated beads recognizing Myc. (D) Upper: USC02 GSCs were transfected with control (shCtrl) or shCK1A shRNA and treated with cycloheximide (CHX) for indicated times (h, hours) before harvest. Lower: HEK293T cells were treated with DMSO or the CK1A inhibitor D4476 (25 $\mu \mathrm{M})$ and treated with CHX for indicated times before harvest. (E and F) Effect of CK1A activation or inhibition on MBD3 protein stability. T98C CSCs were treated with varying doses of pyrvinium pamoate (Pyr-Pam) (E) or 10 $\mu \mathrm{M}$ Pyr-Pam and 0-50 $\mu \mathrm{M} 04476$ as indicated (F). WCLs were immunoblotted with the indicated antibodies. Data are presented as the mean \pm SD and are representative of at least 3 independent experiments. ${ }^{* *} P<0.005$; ${ }^{* *} P<0.0005$ by 2 -way ANOVA with Bonferroni's post hoc test.

proteasomal degradation. Consistently, CK1A activation in the presence of increasing doses of Pyr-Pam increased MBD3 ubiquitination relative to vehicle controls, whereas CK1A inactivation by treatment with $\mathrm{D} 4776$ had no effect on MBD3 ubiquitination in T98G GSCs (Figure 5C). Interestingly, CK1A-mediated MBD3 polyubiquitination was completely abolished in T98G GSCs transfected with the MBD3(SallA) mutant (Supplemental Figure $4 \mathrm{H}$ ), suggesting that phosphorylation of multiple serines in MBD3 by CK1A recruits BTRCP, resulting in polyubiquitination and protein destabilization. Also, following BTRCP depletion, CK1A overexpression did not alter MBD3 polyubiquitination (Figure 5D), indicating that BTRCP functions downstream of CK1A in this context.

$C K 1 A$ and BTRCP/MBD3 signaling regulates GSC self-renewal and activation in GBM. To determine whether CK1A and BTRCP/ MBD3 signaling activates GSCs, we knocked down MBD3 in LN229, U118MG, U87MG, U251MG, and T98G cells, and in T98G and USC02 GSCs and assessed proliferation and selfrenewal capacities (Figure 6, A-F, and Supplemental Figure 5, A-C). MBD3-deficient GBM cells exhibited a significant decrease in both the number and size of foci (Figure 6, A-F, and Supplemental Figure $5 \mathrm{~A}$ ) and a significant decrease in proliferation (Supplemental Figure 5B) relative to shScramble. Secondary sphere-forming capacity, an indicator of self-renewal, also decreased significantly in MBD3-deficient cells relative to shScramble or control GSCs (Supplemental Figure 5C). Interestingly, MBD3-depleted T98G GSCs showed significantly decreased levels of the GSC markers CD133, CD44, and CXCR4 and pluripotency markers OCT4 and SOX2 relative to shScramble cells (Figure 6G). We then performed MTT and sphere-formation assays to compare the effect of CK1A activation or inhibition on GBM cell proliferation and self-renewal. Proliferation was efficiently dose-dependently inhibited following treatment with the CK1A activator Pyr-Pam but not with the CK1A inhibitor D4476 (Figure 7A). Sphere-forming capacity of LN229, U87MG, U118MG, U251MG, and T98G GSCs significantly decreased in Pyr-Pam-treated cells, while we observed no significant effect in D4476-treated cells (Figure 7B and Supplemental Figure 5D). Interestingly, Pyr-Pam-dependent inhibition of secondary sphere-forming capacity was significantly blocked in T98G GSCs stably transduced with lentivirus harboring $M B D 3(\Delta 2 D)$ or MBD3(SallA) mutant constructs (Supplemental Figure 6, A and B), strongly suggesting that CK1A activation inhibits self-renewal and stem cell activity by regulating MBD3 protein stability. These outcomes were confirmed by a dose-dependent decrease in expression of GSC and pluripotency markers in Pyr-Pam-treated cells; 

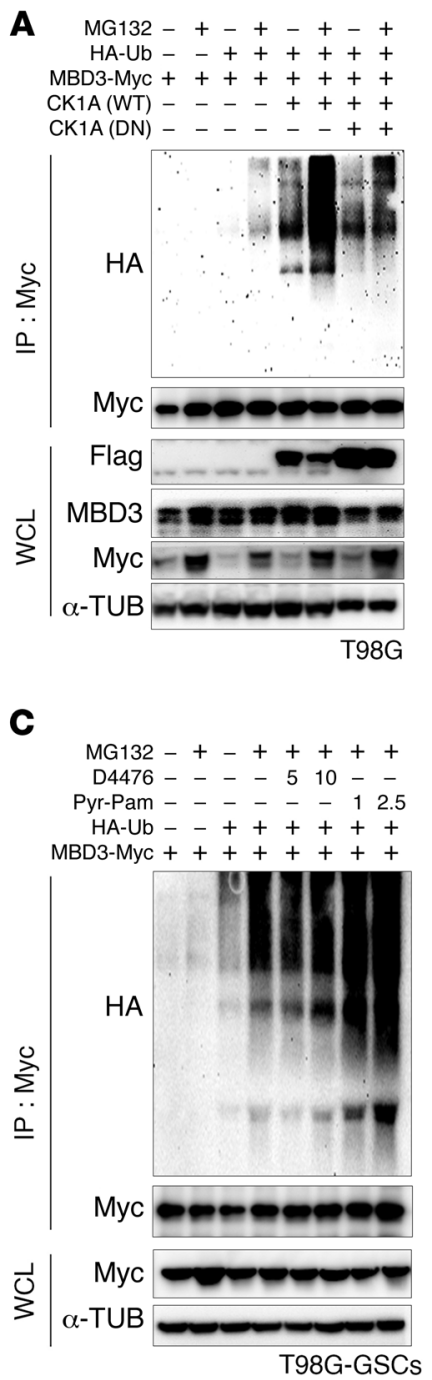
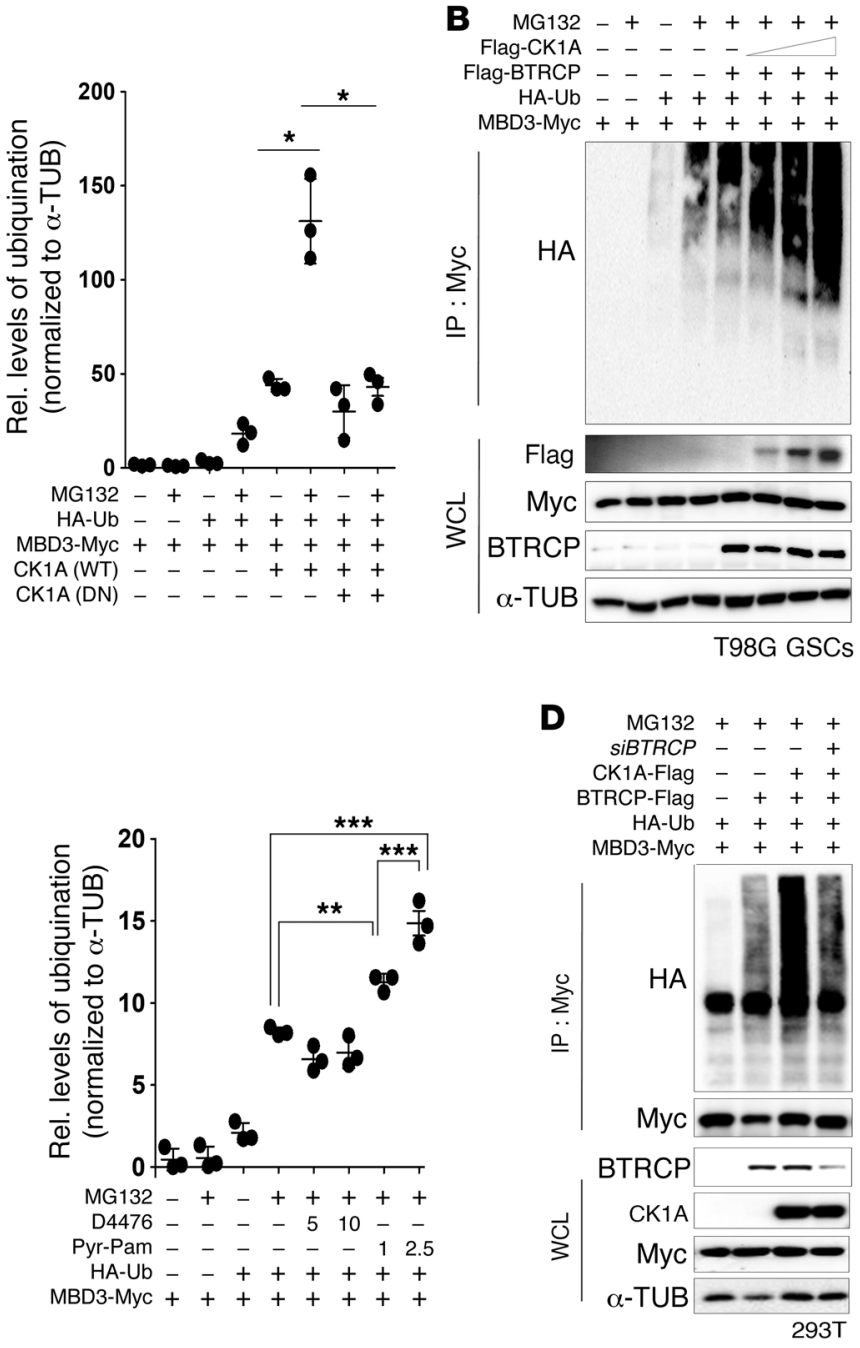
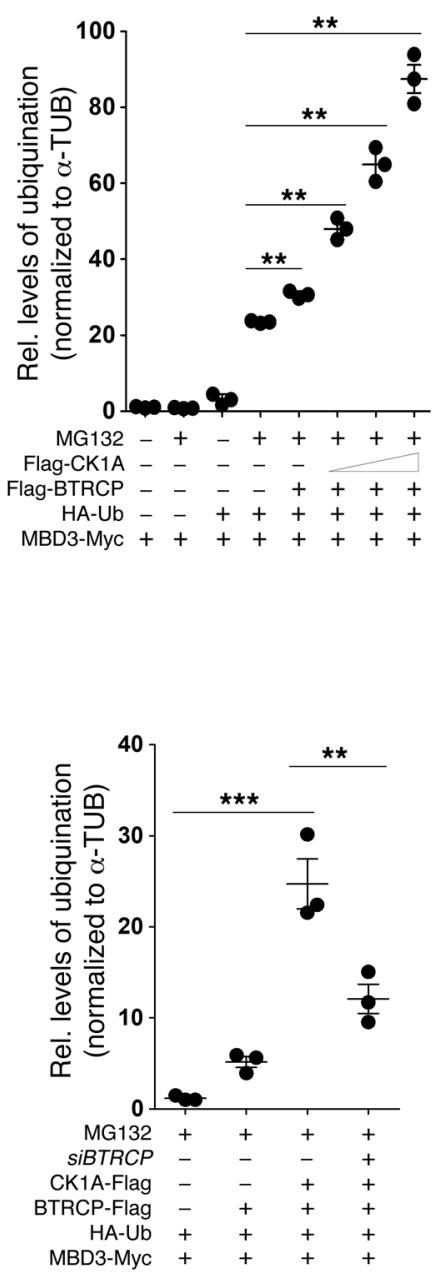

Figure 5. CK1A facilitates BTRCP-mediated MBD3 polyubiquitination. (A-C) Effect of CK1A activation or inhibition on MBD3 polyubiquitination. T98G cells or T98G GSCs were transfected with the indicated vectors and/or treated with varying doses of either D4476 or Pyr-Pam. Whole-cell lysates (WCLs) were then immunoprecipitated with the indicated beads recognizing Myc. Quantification of polyubiquitinated MBD3 normalized to $\alpha$-tubulin ( $n=3$ ). (D) Effect of BTRCP knockdown on CK1A-mediated MBD3 polyubiquitination. HEK293T cells were transfected with the indicated vector and then treated with MC132. WCLs were then immunoprecipitated with the indicated beads recognizing Myc. DN, dominant negative CK1A construct. Data are presented as the mean \pm SD and are representative of at least 3 independent experiments. ${ }^{*} P<0.05$; ${ }^{*} P<0.005$; ${ }^{* *} P<0.0005$ by 1 -way ANOVA with Tukey's multiple-comparison test.

levels of CD133, CD24, CD44, CXCR4, OCT4, NANOG, SOX2, and KLF4 transcripts, as well as those of the NPC markers NESTIN, PAX6, and BMI1, in T98G GSC spheres significantly decreased following Pyr-Pam treatment (Figure 7C).

TMZ is the standard drug used to treat patients with GBM, and TMZ chemoresistance positively correlates with epigenetic silencing of the $O^{6}$-methylguanine-DNA methyl-transferase (MGMT) gene and with the presence of GSCs $(36,37)$. We observed significantly higher MGMT expression in glioma samples from highgrade (stages III and IV) than in low-grade tumors (stage II) (Supplemental Figure 6C). Moreover, high MGMT gene expression was also positively correlated with GSC marker expression and that of BCL3 and IDH2, both known to influence GBM sensitivity to TMZ (refs. 38-40 and Supplemental Figure 6C).

To assess whether Pyr-Pam treatment can overcome chemoresistance in GBM expressing high levels of MGMT, we first compared the effect of TMZ, Pyr-Pam, or cotreatment with both using LN229, U118MG, and U87MG glioma cells, which show low MGMT levels, and U251MG and T98G cells, which show high MGMT levels (Figure 7D and Supplemental Figure 6D). TMZ or Pyr-Pam inhibited LN229, U118MG, and U87MG growth, and low concentrations of Pyr-Pam alone significantly decreased the number and size of those GBM foci compared with vehicle- or TMZonly-treated cells (Figure 7D). However, TMZ had no inhibitory effect on U251MG and T98G cell growth (Figure 7D). To assess cytotoxic effects of Pyr-Pam on TMZ-resistant GBM cells or GSCs, we performed MTT and colony-forming assays using 10 different human GBM lines (LN229, LN229-TMZ resistant, U118MG, U118MG-TMZ resistant, U87MG, U87MG-TMZ resistant, T98G, T98G-TMZ resistant, U251MG, and U251MG-TMZ resistant) and 2 different GSC lines (T98G GSCs and USC02). That analysis showed that Pyr-Pam significantly inhibited proliferation and 

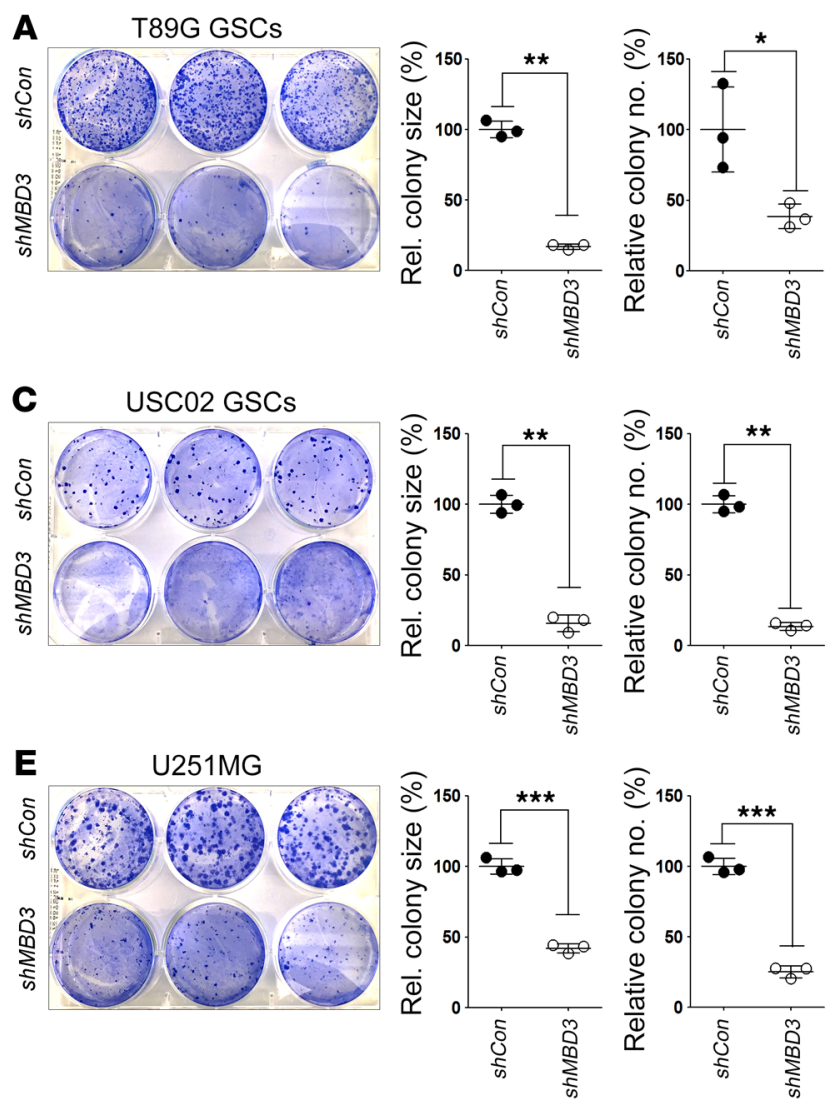
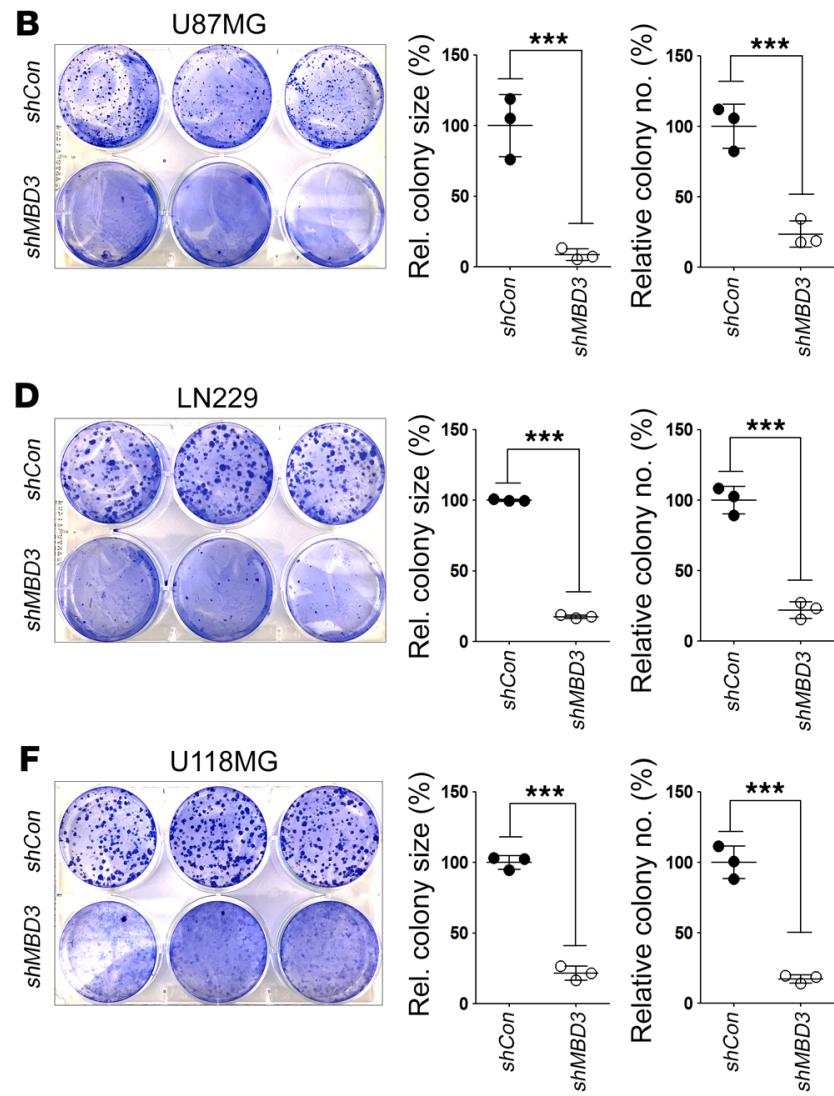

G
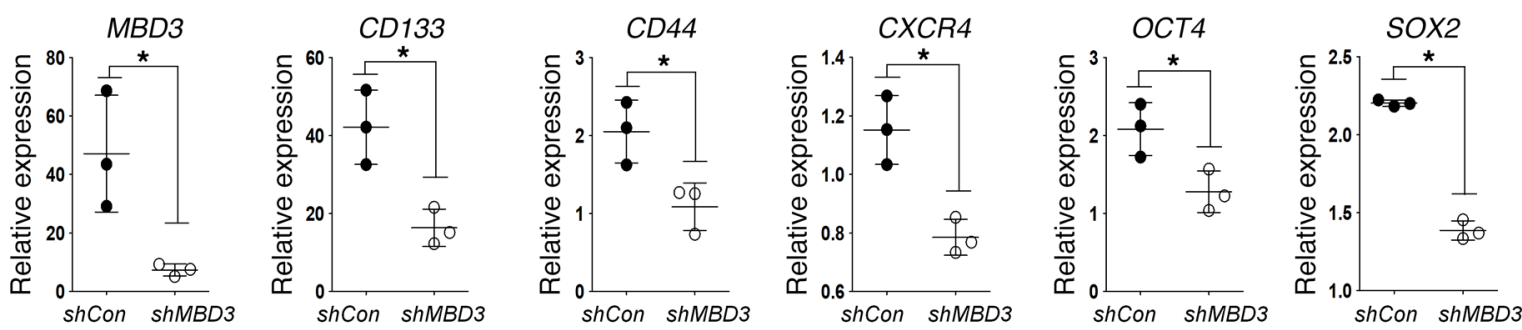

Figure 6. MBD3 loss inhibits GBM proliferation and stemness activity in vitro. (A-F) T98G GSCs or USC02 GSCs infected with shScramble or shMBD3 lentivirus were seeded on 6-well plates and assayed for colony formation. Foci were photographed (left) and quantified (right). (C) qPCR analysis to detect the indicated mRNAs. ${ }^{*} P<0.05 ;{ }^{* *} P<0.005 ;{ }^{* *} P<0.0005$ by 2-way ANOVA with Bonferroni's post hoc test (A-F) or unpaired 2-tailed Student's $t$ test (G).

colony-forming activity of both TMZ-sensitive and -resistant GBM cells or GSCs (Supplemental Figure 7, A-I). Collectively, these results suggest that chemoresistance is correlated with high MGMT expression, and that Pyr-Pam overcomes resistance to TMZ.

$M B D 3$ loss inhibits GBM progression by eradicating GSCs in vivo. To assess in vivo effects of MBD3 depletion on GBM progression, we used U118MG-pLuc-GFP cells, which express low MGMT levels, or U251MG-pLuc-GFP cells, which express high MGMT levels. Note that both lines express luciferase and GFP for purposes of xenograft and luminescence imaging. We first infected both U118MG and U251MG with shScramble or shMBD3 lentivirus and then intracranially injected each into immunocompromised mice to evaluate tumor formation. Injection of shScramble cells of either line elicited tumorigenesis within 3 weeks. However, mice injected with either line transduced with $s h M B D 3$ did not show detectable tumor growth (Figure 8, A-D). We then asked whether inhibition of tumor growth following $M B D 3$ knockdown is associated with GSC inactivation by quantifying stained GSC populations in xenograft tumors. Interestingly, expression levels of CD133, CD44, CXCR4, MBD3, nestin, and Ki67 markedly decreased in shMBD3-transduced U118MG- and U251MG-pLuc-GFP xenografts relative to xenografts injected with corresponding shScramble cells (Figure 8, E-J), suggesting that MBD3 loss inhibits progression of human GBM in vivo by promoting loss of GSCs.

Next, to investigate in vivo effects of Pyr-Pam treatment in GBM or GSCs expressing high MGMT levels, we intracranially injected U118MG-, U251MG-, and T98G-pLuc-GFP GSCs (i.c. model), or subcutaneously injected patient-derived primary and recurrent GBM cells (s.c. model) into immunocompromised mice. After 4 days, we implanted a micro-osmotic pump to automatically infuse vehicle, TMZ, or Pyr-Pam $(0.11 \mu \mathrm{L} / \mathrm{h})$ into the brain for the i.c. model and administered those drugs intratumorally 
A

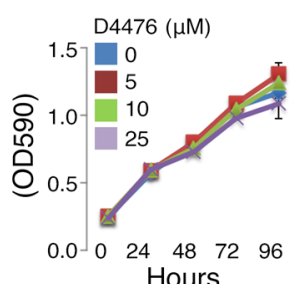

Hours

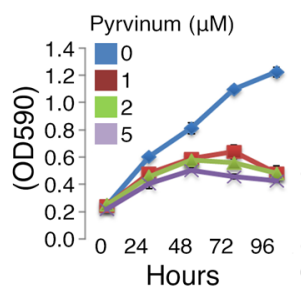

Hours
B
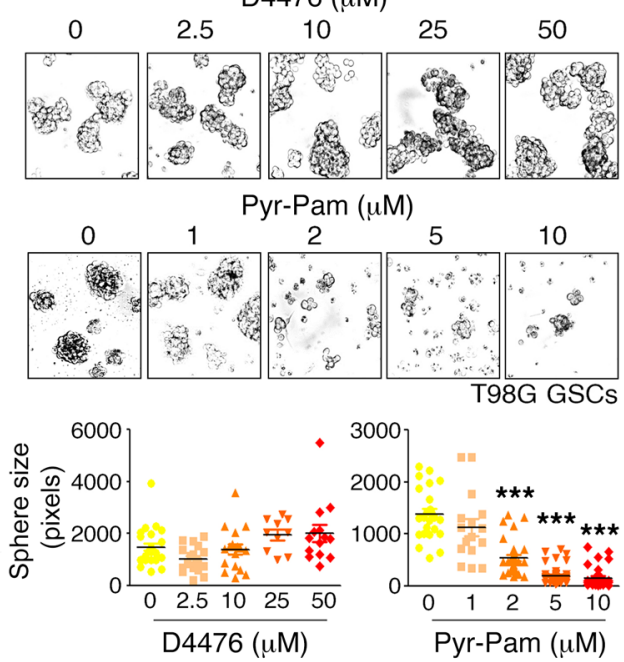

C
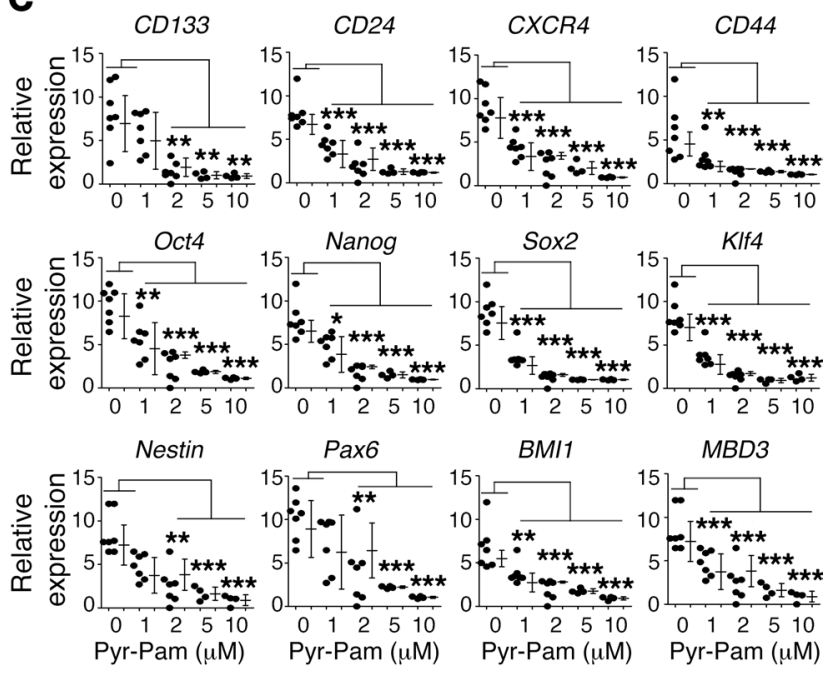

D
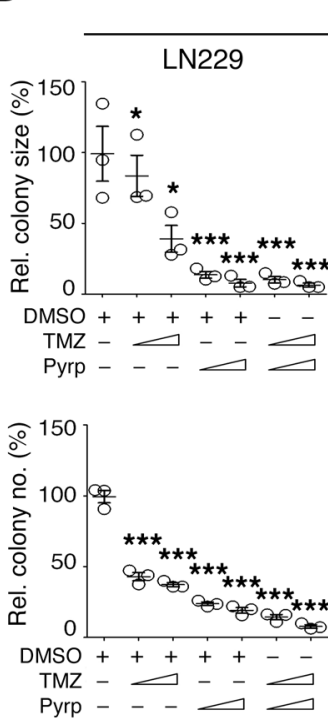

MGMT Iow
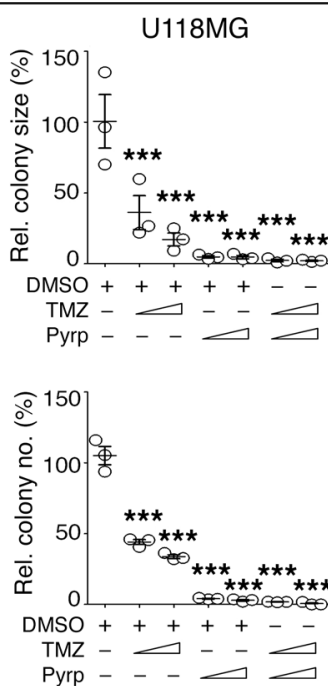
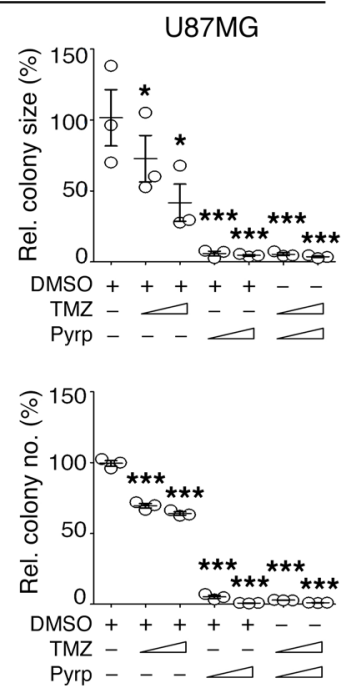

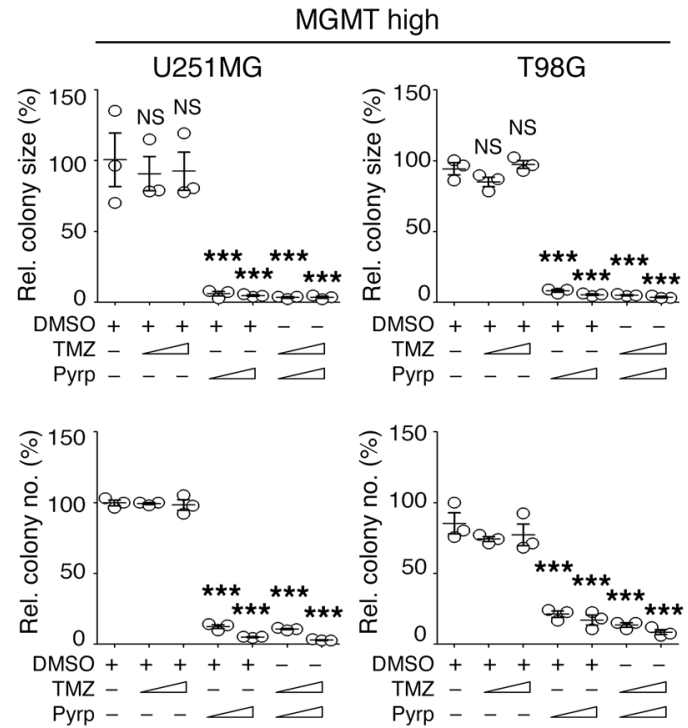

Figure 7. CK1A activation inhibits GBM cell proliferation in vitro. (A) T98G cells were treated with the indicated concentrations of D4476 or Pyr-Pam for 96 hours, and proliferation was quantified using an MTT assay. (B) T98G GSCs were seeded at a density of $2 \times 10^{4}$ cells/plate in ultra-low-attachment 96-well plates and then treated with the indicated concentrations of D4476 or Pyr-Pam for 3 days to allow sphere formation. Lower panel: Relative size of spheres of each group was measured by Imagel software. (C) qPCR analysis of spheres shown in B to detect the indicated mRNAs. (D) LN229, U118MG, and U87MC (MGMT-low expressing) or U251MG and T98C (MGMT-high expressing) cells were seeded on 12-well plates and treated with the indicated concentrations of TMZ, Pyr-Patm, or both for 8 days. Colony-formation assays were performed. Foci were quantified using Imagel software. Rel., relative; TMZ, temozolomide; Pyrp, Pyr-Pam (pyrvinium pamoate). Data are presented as the mean \pm SD and are representative of at least 3 independent experiments. ${ }^{*} P<0.05$; ${ }^{* *} P<0.005 ;{ }^{* *} P<0.0005$ by 1-way ANOVA with Tukey's multiple-comparison test.

for the s.c. model (Figure 9, A-D, Supplemental Figure 8, A and B, Supplemental Figure 9, A-L, and Supplemental Table 5). We then measured tumor volume over time by in vivo imaging. Tumors of both the i.c. model and the s.c. model treated with vehicle elicited rapid tumorigenesis within 4 weeks of injection. TMZ treatment had a significantly inhibitory effect on the growth of xenograft tumors derived from U118MG-pLuc-GFP cells, which express low MGMT levels, and a slight inhibitory effect on the 2 different patient-derived primary GBM cells. However, TMZ treatment did not have an effect in the context of U251MG- and T98G-pLucGFP GSCs and recurrent human GBM cells (Figure 9, B and D, and Supplemental Figure 9, B, F, and J). However, none of the GBM cells treated with Pyr-Pam showed detectable tumor growth over time (Figure 9, B and D, Supplemental Figure 8B, and Supplemental Figure 9, A, B, E, F, and J). Moreover, the number of $\mathrm{MBD}^{+}, \mathrm{CD} 133^{+}, \mathrm{CD}_{4} 4^{+}, \mathrm{CXCR}^{+}$, nestin $^{+}$, and $\mathrm{Ki} 67^{+}$cells markedly decreased in Pyr-Pam-treated GBM xenografts relative to corresponding samples treated with vehicle or TMZ (Figure 9, E-J, and Supplemental Figure 9, C, G, K, and L). Additionally, Pyr-Pam inhibition of sphere-forming capacity and tumorigenesis was significantly blocked in MBD3( $\triangle 2 D$ )- and MBD3(SallA)mutant T98G cells but not in T98G cells expressing WT MBD3 
A
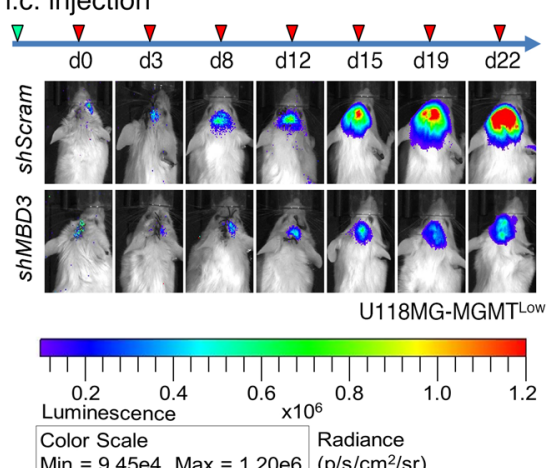

Min $=9.45 \mathrm{e} 4, \operatorname{Max}=1.20 \mathrm{e} 6\left(\mathrm{p} / \mathrm{s} / \mathrm{cm}^{2} / \mathrm{sr}\right)$

B

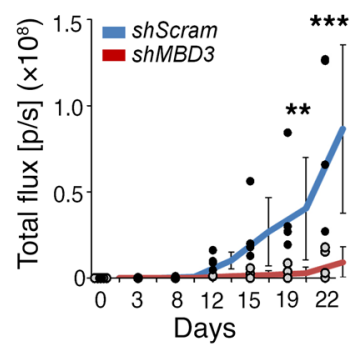

C i.c. injection

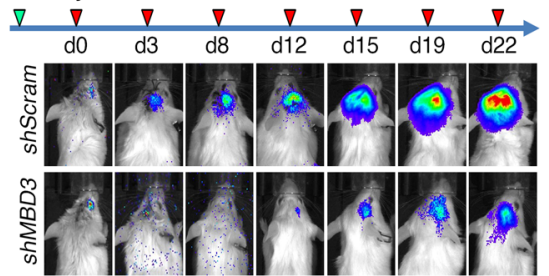

U251MG-MGMTHigh
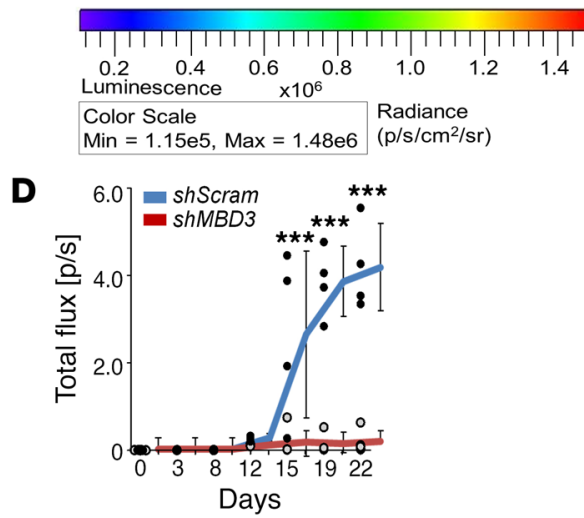

E

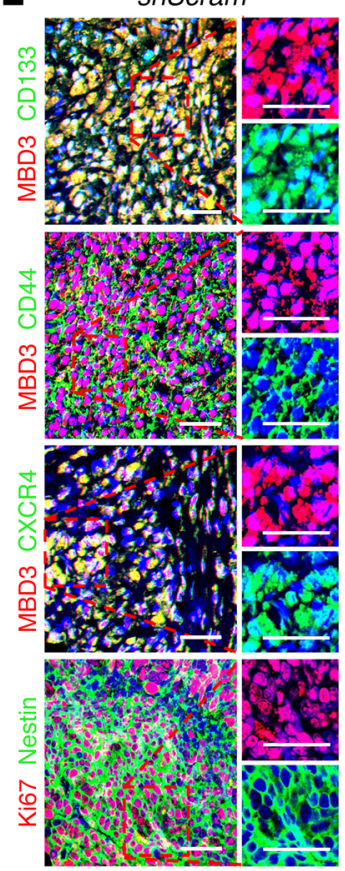

H

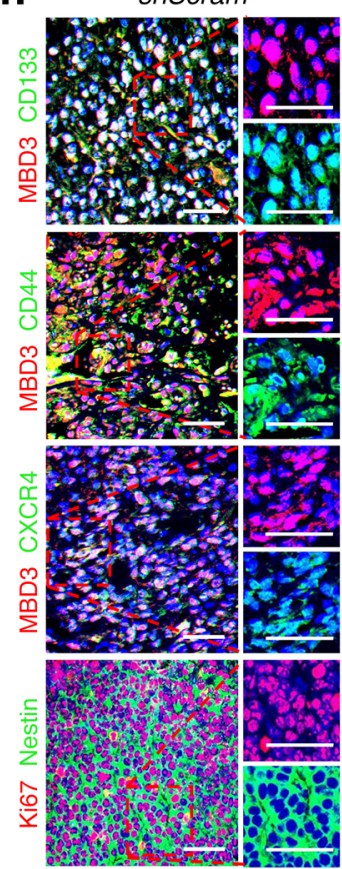

$\mathbf{F}$
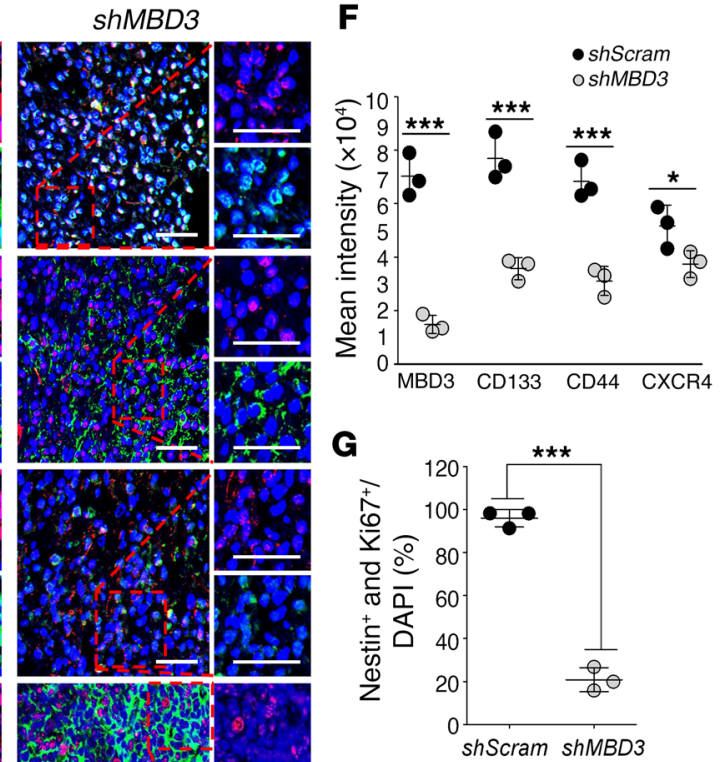

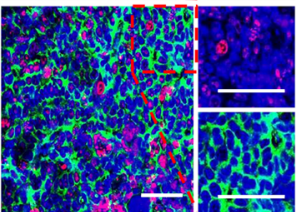

U118MG-MGMTLow

shMBD3
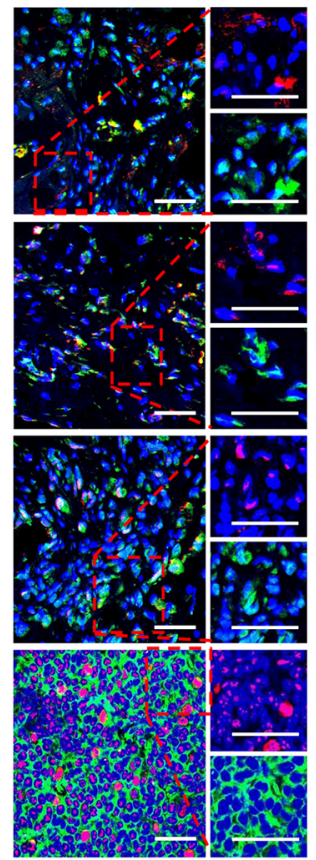

U251MG-MGMTHigh

\section{I}
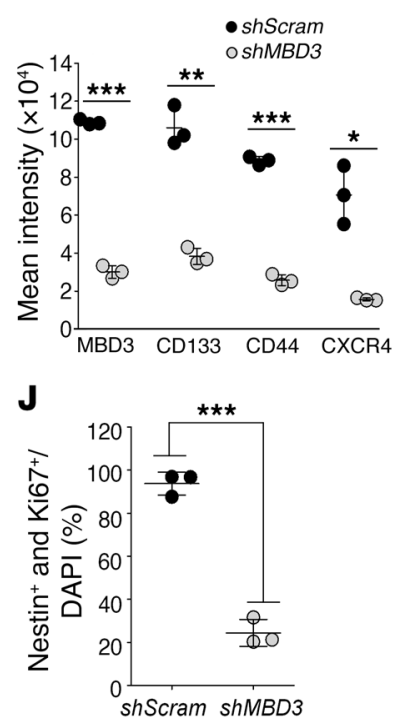

Figure 8. MBD3 loss inhibits progression of GBM with either low or high MGMT expression. (A-J) pLuc-U118MG cells or pLuc-U251MG cells depleted of MBD3 by shMBD3 or shScramble lentiviral controls were intracranially injected into immunocompromised (NSG) mice. (A-D) Serial bioluminescence imaging was used to monitor tumor volume (each group, $n=5$ ). (B and $\mathbf{D}$ ) Tumor volume was measured every 3 or 4 days. Quantification (total flux; $p / s$, photons per second) of the bioluminescent signal from tumor regions in $\mathbf{A}$ and $\mathbf{C}$. (E-J) After 4 weeks, mice were sacrificed and analyzed immunohistochemically with the indicated antibodies. Expression of CD133, CD44, CXCR4, and MBD3 (F and I) or Ki67 and nestin (G and J) was quantified using Image) software. Images were captured using a Zeiss confocal microscope. Representative images were selected from at least 3 different fields. Data are presented as the mean $\pm \mathrm{SD} .{ }^{*} P<0.05 ;{ }^{* *} P<0.005 ;{ }^{* *} P<0.0005$ by 2-way ANOVA with Bonferroni's post hoc test (B and $\mathbf{D}$ ) or unpaired, 2 -tailed Student's $t$ test (F, G, I, and J). Scale bars: $50 \mu \mathrm{m}$; and inset scale bars: $50 \mu \mathrm{m}$. 
(Supplemental Figure 10, A-D), strongly suggesting that the effect of Pyr-Pam treatment on self-renewal and tumorigenesis is associated with its effects on MBD3 stability.

$M B D 3$ loss or CK1A activation blocks enrichment of the MBD3NuRD complex on neuronal and glial gene loci and promotes GSC differentiation. We recently showed that MBD3 functions as an epigenetic regulator in NPCs by recruiting NuRD complex components such as HDAC1, HDAC2, and MTA1 to gene loci associated with neurogenesis $(12,13)$. Others have shown that MBD3 is enriched at promoters, gene bodies, and enhancers of active genes in breast cancer whose gene expression overlaps with that of neuronal cells (41). Accordingly, here we found that human MBD3 specifically binds proximal promoter regions and gene bodies of neuronal or glial genes such as DLX1, TUJ1, LBX1, and GFAP (Supplemental Figure 11A and Supplemental Table 6). We then asked whether MBD3 binds corresponding loci in GBM cells. To do so, we undertook ChIP-qPCR with an anti-MBD3 antibody in T98G, U87MG, and LN229 GBM cells and confirmed specific enrichment of MBD3 on DLX1, TUJ1, LBX1, and GFAP gene loci in those cells (Supplemental Figure 11, B-E). Because MBD3 represses transcription by recruiting the NuRD complex to target loci $(12,13)$, we undertook ChIP-qPCR with antibodies against NuRD complex components MBD3, HDAC1, HDAC2, and MTA1 and against acetyl-histone $\mathrm{H} 3$, as a marker of active gene expression, in either shScramble- and shMBD3-transduced GBM cells cultured in differentiation conditions (Figure 10, A-D). ChIP-qPCR analysis revealed that enrichment of HDAC1, HDAC2, and MTA1 on target gene loci significantly decreased in $s h M B D 3$ relative to shScramble GBM cells (Figure 10, A and B), while levels of acetylhistone $\mathrm{H} 3$ increased (Figure 10, C and D). Moreover, we did not observe decreases in NuRD complex components in shMBD3 compared with shScramble GBM/GSCs (Supplemental Figure 12A). These findings suggest that MBD3 depletion inhibits NuRD recruitment to neuronal or glial cell differentiation-associated gene loci and increases acetyl-histone $\mathrm{H} 3$ activity and transcription during differentiation.

Next, to determine how Pyr-Pam blocks GBM tumor progression and GSC self-renewal, we performed ChIP-qPCR with MBD3, HDAC1, HDAC2, MTA1, and acetyl-histone $\mathrm{H} 3$ antibodies using GBM cells or USC02 GSCs in differentiation conditions and treated them with Pyr-Pam or DMSO vehicle (Figure 11, A-D, and Supplemental Figure 12, B and C). ChIP-qPCR analysis revealed that enrichment of HDAC1, HDAC2, and MTA1 at target gene loci significantly decreased in Pyr-Pam-treated GBM cells or USCO2 GSCs relative to DMSO-treated cells (Figure 11, A and B, and Supplemental Figure 12B), while levels of acetyl-histone $\mathrm{H} 3$ increased (Figure 11, C and D, and Supplemental Figure 12C). These findings suggest that Pyr-Pam-mediated MBD3 degradation inhibits recruitment of NuRD components to neuronal or glial cell differentiation gene loci and increases histone $\mathrm{H} 3$ acetylation, leading to transcriptional activation of these target genes (Figure 11E).

\section{Discussion}

In this study, we identify a tumor-initiating subpopulation of GBM cells with GSC potential and demonstrate that MBD3 protein stability regulated by $\mathrm{CK} 1 \mathrm{~A} / \mathrm{BTRCP}$ signaling regulates GSC activity and GBM progression and recurrence. Using RNA sequencing and immunohistochemical analysis in combination with sphere-formation and xenograft analyses, we observed that $\mathrm{CD} 133^{+} \mathrm{CD} 44^{+} \mathrm{CXCR} 4^{+}$triple-positive cells have higher selfrenewal and tumor-forming capacity than single- or doublepositive or triple-negative cells. Like normal NPCs, these GSC subpopulations are multipotent and can give rise to both neuronal and glial cells. Importantly, we found that MBD3 protein levels correlate positively with GSC marker expression, and that MBD3 protein becomes less stable as GSCs differentiate. Using functional analysis in GBM cell lines, we show that MBD3 overexpression enhances GSC marker expression and, conversely, that MBD3 loss significantly decreases marker expression. At the same time, we observed decreased sphere-forming activity in MBD3-depleted GBM cells as well as decreased tumor growth in xenograft mice injected with MBD3-depleted GBM cells, suggesting that MBD3 could be a drug target for GBM treatment.

MS analysis and co-IP experiments reported here also identified SKP1, CUL isoforms, and the F-box protein BTRCP as MBD3-interacting partners in GBM cells. Substrate-recognition components of the SKP1-CUL1-F-box-protein (SCF) complex are important players in many mammalian biological functions (29). BTRCP is a known E3 ligase that recognizes and ubiquitinates phosphorylated targets, promoting their degradation (31). So far, several BTRCP substrates have been reported, including $\beta$-catenin, Emil, and inhibitor of nuclear factor- $\kappa \mathrm{B}$ (IкB), and many function in signal transduction and cell cycle regulation in cancer cells (42). MBD3 is an epigenetic substrate of BTRCP. This finding is of particular interest, as aberrant MBD3 expression and subsequent epigenetic changes may cause developmental defects as well as cancers $(13,30)$.

We also used kinase-specific phosphorylation site prediction software to determine which kinase(s) is responsible for MBD3 phosphorylation at candidate serines $39,45,85$, and 106 and identified CK1A. CK1A functions in cell cycle regulation, apoptosis, and DNA repair, and is also considered a tumor suppressor $(33,43$, 44). Our finding that CK1A phosphorylates MBD3 serine residues recognized by BTRCP reveals what we believe is a novel MBD3 degradation pathway and demonstrates that CK1A-triggered, BTRCP-dependent MBD3 degradation regulates GSC activity. Importantly, CK1A activation by the allosteric activator Pyr-Pam in GSCs or recurrent GBMs promoted GSC differentiation by blocking enrichment of the repressive MBD3-NuRD complex on neural differentiation gene loci and therefore inhibited tumorigenesis (45).

Finally, our observation that MBD3 inhibits neural differentiation of GSCs but maintains GSC self-renewal is somewhat discrepant from observations reported by Cui et al. (46), who showed that MBD3 expression is lower in human GBM than in normal brain, and that MBD3 loss promotes brain tumor proliferation and migration. The study by Cui et al., however, analyzed the SF767 GBM line, which may account for the discrepancy. The SF767 line reportedly shows DNA fingerprinting identical to that seen in the squamous cell carcinoma line ME-180 (47). There are also reports that phenotypes seen in SF767 cells vary widely compared with other gliomas, specifically in terms of morphology of tumors grown in mice (48) and patterns of E-cadherin expression (49). Further studies are needed to assess the relevance of the primary SF767 cell line to our GBM studies. We also note that our findings relevant to MBD3 function are consistent across several commercially available GBM cell lines 
A

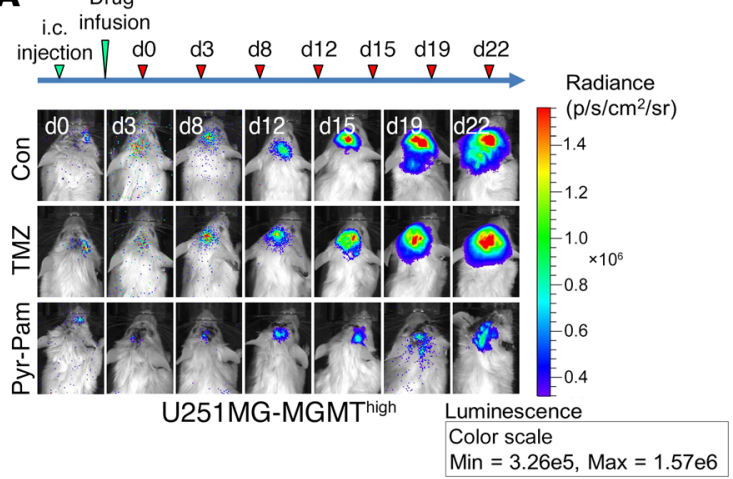

E

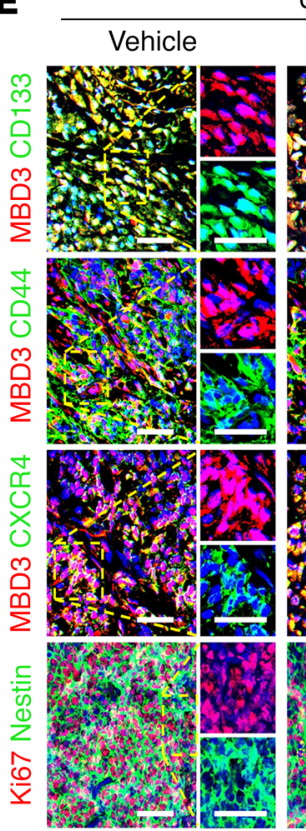

U251MG-MGMThigh
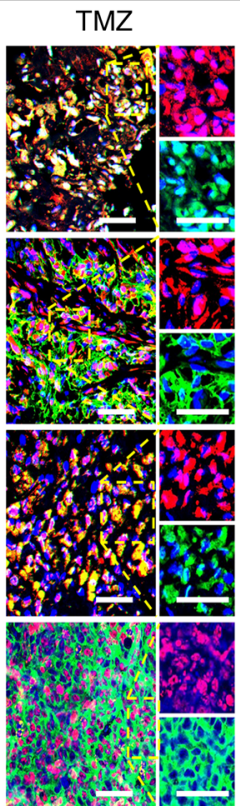

H

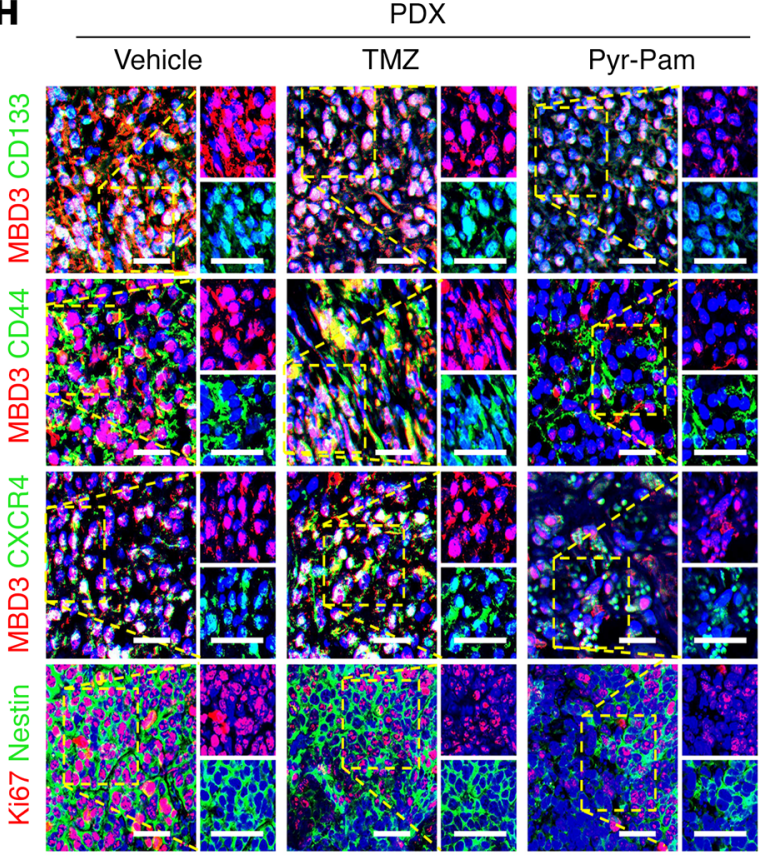

B

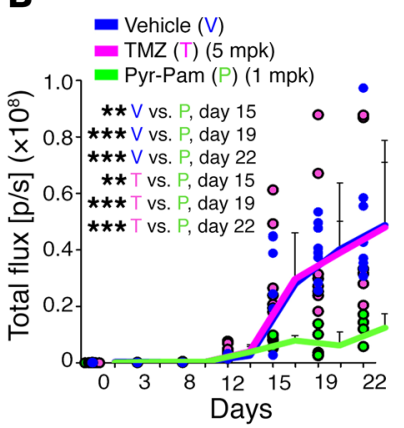

TMZ (T) $(5 \mathrm{mpk})$

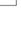

presem

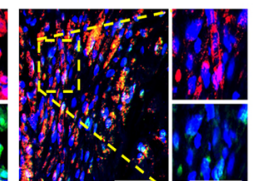
F $\bullet$ veh
OTMZ (5 mpk)
OPyr-Pam (1 mpk)
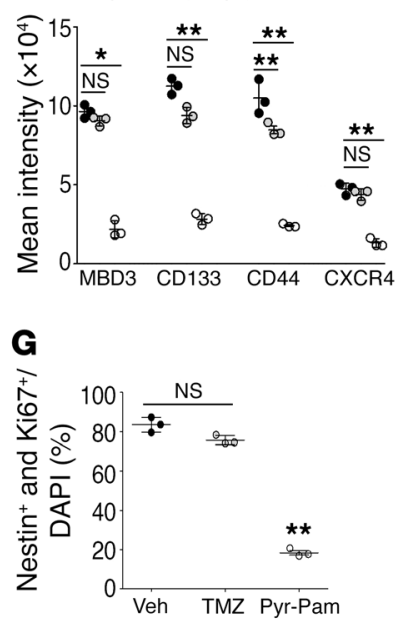

Cells $\left(1 \times 10^{6}\right)$ Measurement of volumes

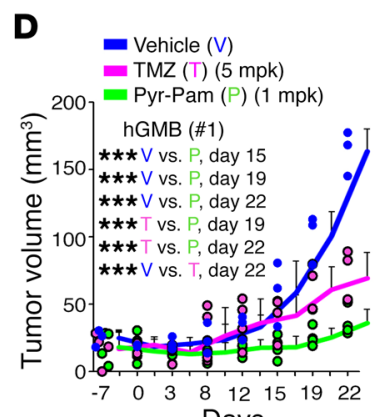

Days

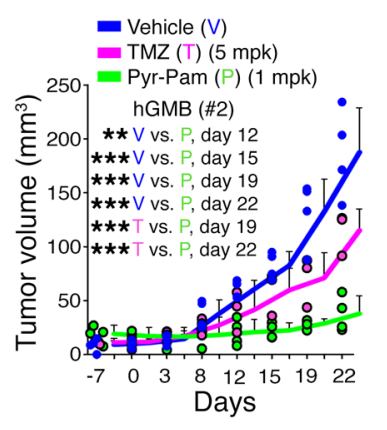

Figure 9. Stimulation of CK1A signaling inhibits progression of TMZ-resistant CBM with high MGMT expression and patientderived primary GBM. (A and C) pLucU251MG cells or human primary CBM cells were intracranially (i.c.) or subcutaneously (s.c.) injected into immunocompromised (NSG) mice ( $n=4$ or 5 per group). (A) Upper: Schematic showing injection of CBM cells and intrathecal infusion of drugs (vehicle, TMZ [5 $\mathrm{mg} / \mathrm{kg}$ per day], Pyr-Pam [1 mg/kg per day]) for 22 days using implanted osmotic pump. Lower: Representative bioluminescence images of mice at indicated times after intracranial injection. (B) Quantification (total flux; $\mathrm{p} / \mathrm{s}$, photons per second) of the bioluminescent signal from tumor regions shown in A. mpk, $\mathrm{mg} / \mathrm{kg}$. (D) Tumor volumes of Supplemental Figure $8, A$ and $B$, were measured every 3 or 4 days. Tumor volumes were measured using Vernier calipers, applying the formula $\pi / 6 \times$ length $\times$ width $\times$ height. (E-J) After 4 weeks, mice were sacrificed and analyzed immunohistochemically with indicated antibodies. Expression of CD44, CD133, CXCR4, MBD3 (F and I), Ki67, and nestin ( $\mathbf{G}$ and J) in the immunostained CBM tissues in each representative xenograft CBM tumor was quantified using Imagej software. Images were captured using a Zeiss confocal microscope. Representative images were selected from at least 3 different fields. Pyr-Pam, pyrvinium pamoate. Data are presented as the mean $\pm \mathrm{SD}$. ${ }^{*} P<0.05 ;{ }^{* *} P<$ $0.005 ;{ }^{* *} P<0.0005$ by 2 -way ANOVA with Bonferroni's post hoc test (B and $\mathbf{D}$ ) or 1-way ANOVA with Tukey's multiple-comparison test (F, G, I, and J). Scale bars: $50 \mu \mathrm{m}$; and inset scale bars: $50 \mu \mathrm{m}$. 
A

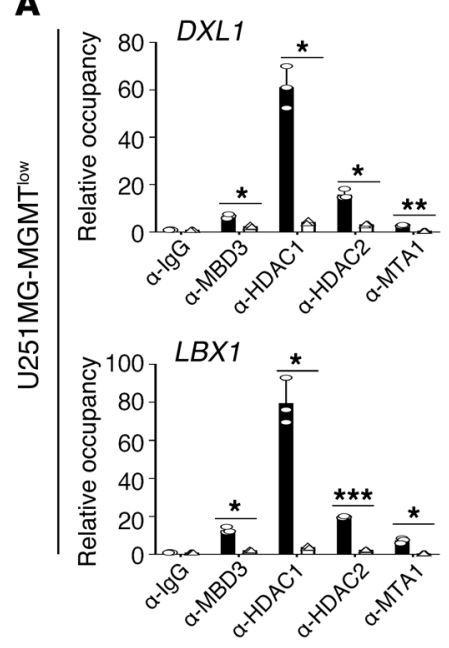

C

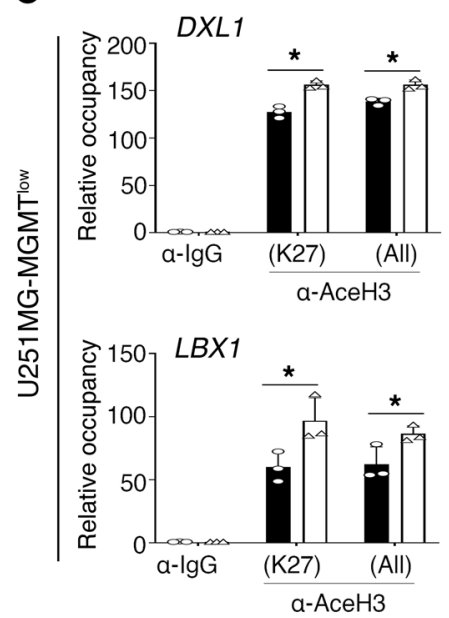

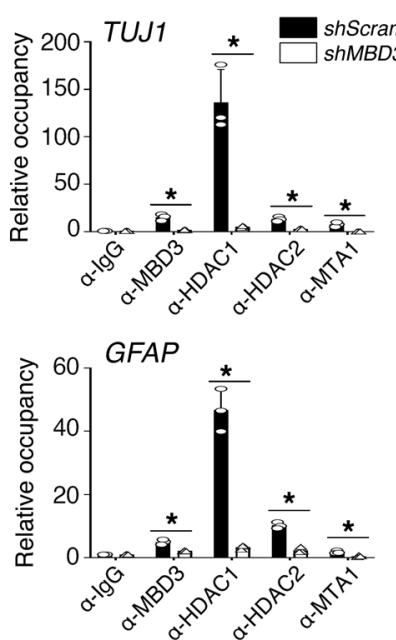
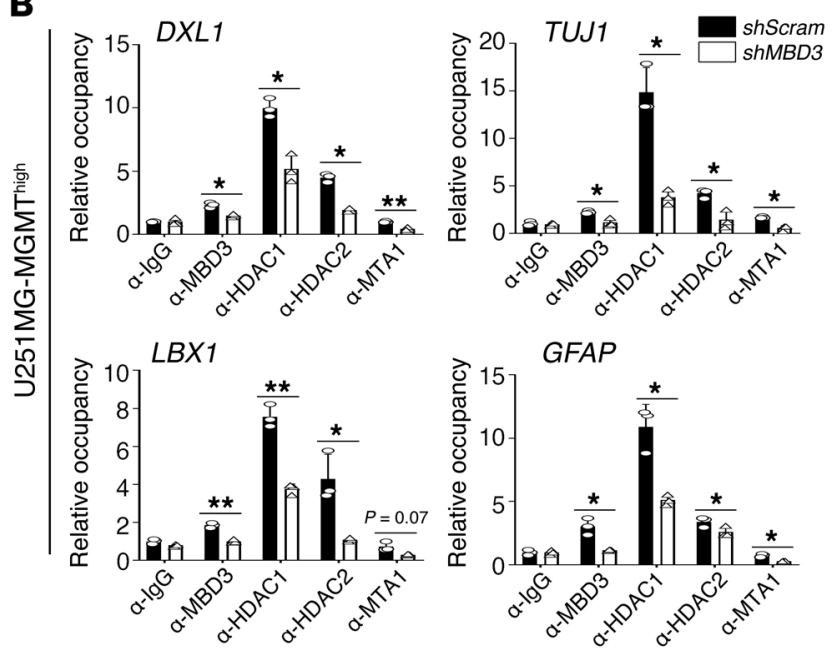

D
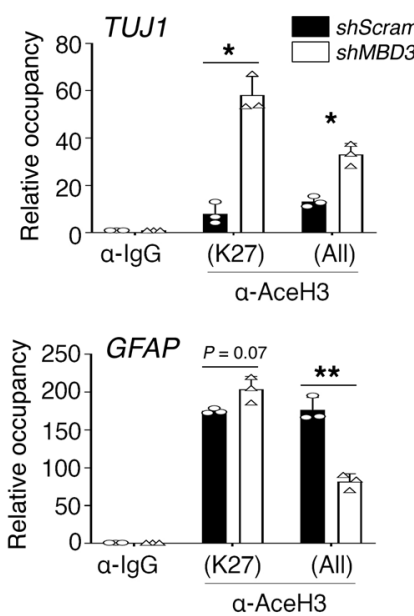

D

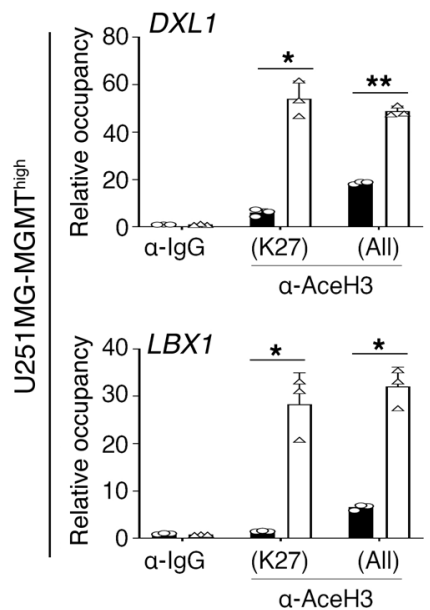

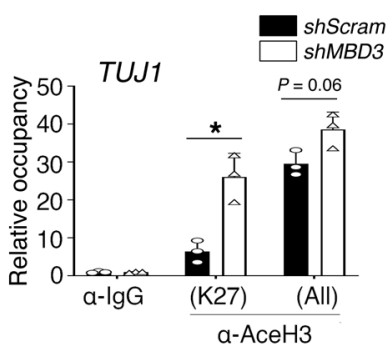

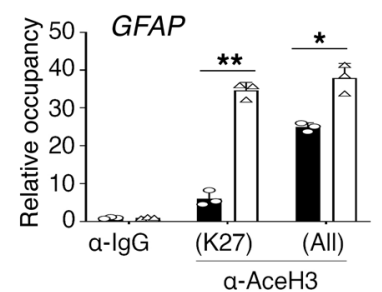

Figure 10. MBD3 loss decreases recruitment of MBD3-NURD transcription repressive complex components and increases acetyl-histone $\mathrm{H3}$ activity on neural differentiation-associated gene promoters. (A-D) ChIP-qPCR analysis of MBD3, HDAC1, HDAC2, MTA1, and acetyl-histone H3 occupancy at MBD3-binding locus in U118MG or U251MG cells treated with shScramble $(n=3)$ and shMBD3 $(n=3)$ lentiviral vectors. Immunoglobulin G (IgG) ChIP served as a negative control. Values are normalized to input control and represent the mean $\pm \mathrm{SD}$. ${ }^{*} P<0.05 ;{ }^{* *} P<0.005 ;{ }^{* * *} P<0.0005$ by unpaired 2-tailed Student's $t$ test.

and in the patient-derived mesenchymal GSC line USC02. Use of this mesenchymal USC02 GSC line and the $\mathrm{CD} 133^{+} \mathrm{CD} 44^{+} \mathrm{CXCR} 4^{+}$ triple-positive population supports our conclusion that BTRCP/ MBD3 signaling could be targeted to eradicate GSCs in GBM.

In summary, we show that $\mathrm{CD} 133^{+} \mathrm{CD} 44^{+} \mathrm{CXCR} 4^{+}$triplepositive GSCs likely contribute to tumor chemoresistance and recurrence and are regulated through the MBD3/BTRCP/CK1A signaling. MBD3 degradation in the context of GSCs requires MBD3 phosphorylation by CK1A on $2 \mathrm{~N}$-terminal degron motifs. Moreover, our work confirms that GSCs and NPCs share mechanisms governing MBD3 protein stability, suggesting that comparable epigenetic pathways operate in both. Finally, we reveal a means to target the CK1A/BTRCP/MBD3 pathway in order to overcome GBM chemoresistance, a strategy that could lead to novel therapies to eradicate these lethal tumors. Given that numerous cancer cells express MBD3, targets identified here are also likely relevant to other types of tumor-initiating cells.

\section{Methods}

Analysis of RNA sequencing data set and screening for putative GSC markers. We analyzed the RNA sequencing data set published in our previous study (32), which consists of 20 normal brain and 19 GBM samples to identify putative GSC markers (Supplemental Dataset 1). We also analyzed an RNA sequencing data set (accession number: GSE48865) that consists of 272 patient samples containing different stages of gliomas (stage II, $n=87$; stage III, $n=47$; stage IV, $n=79$; recurrent, $n=59$ ) (50). Tumor tissue characteristics are summarized in Supplemental Table 1. Potentially novel GSC markers identified are listed in Supplemental Table 2.

Screening for putative GSC markers: patient tissues, brain tumor xenografts, drug infusion, and tissue preparation. For xenograft experiments, immunocompromised (NSG) mice were anesthetized with an Avertin stock solution ( $240 \mathrm{mg} / \mathrm{kg}$; MilliporeSigma) and placed in a stereotaxic apparatus. A midline incision was made using aseptic surgical techniques and the scalp opened to expose the frontal and temporalis 
A

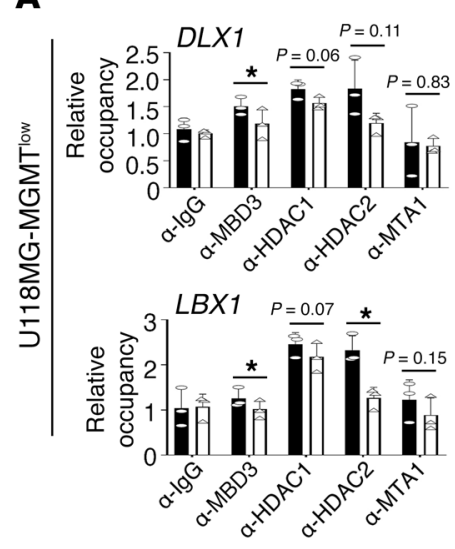

C
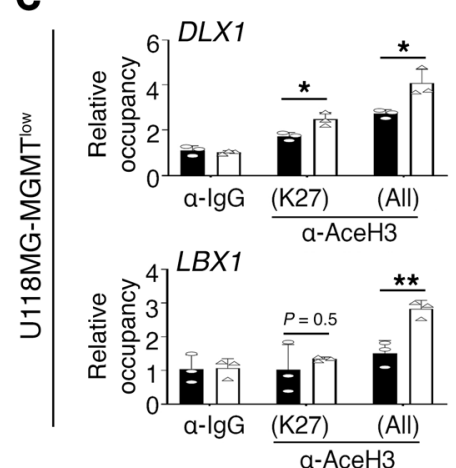
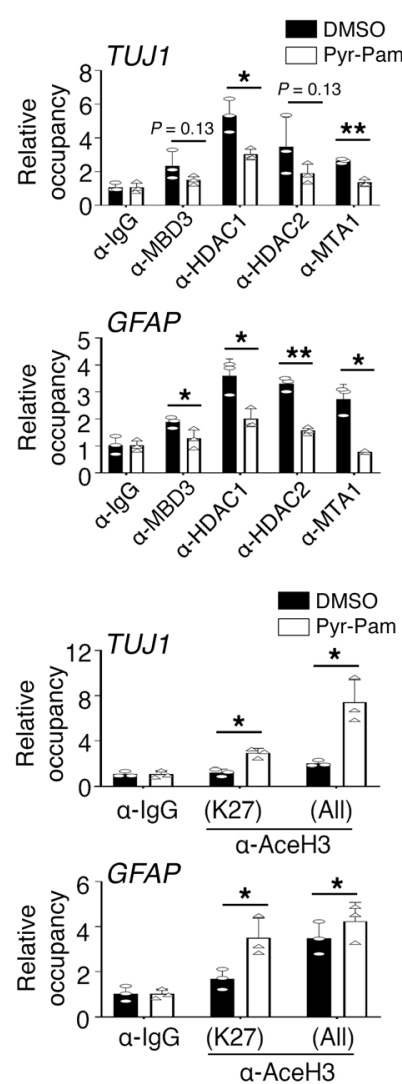

B
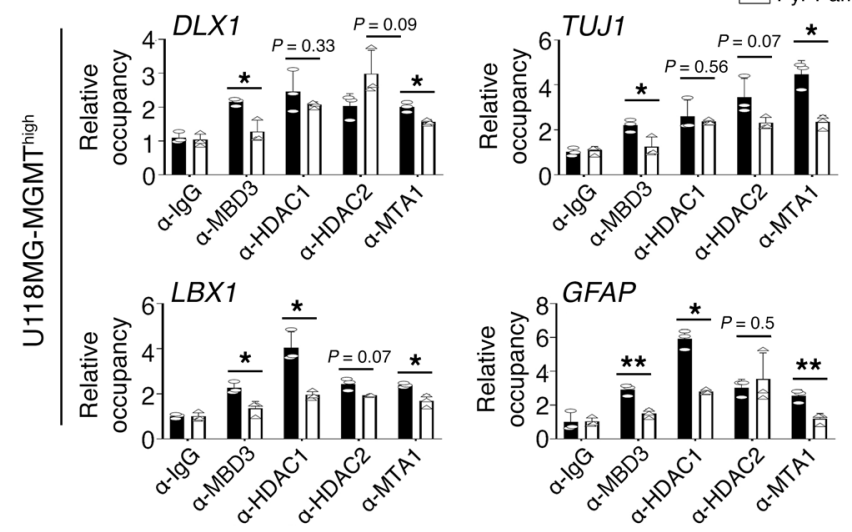

D
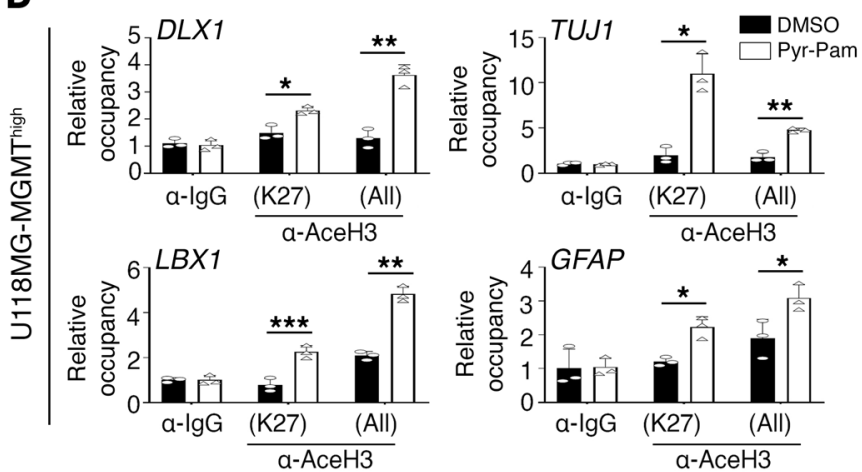

E

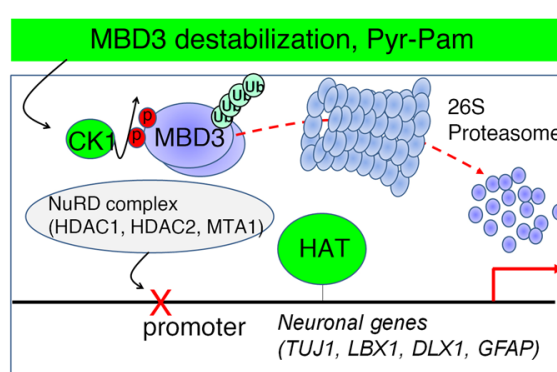

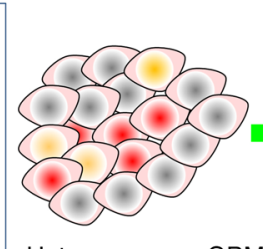

Heterogeneous GBM

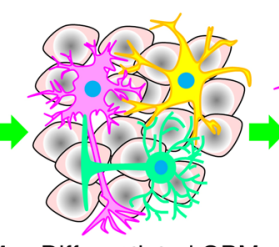

Differentiated GBM

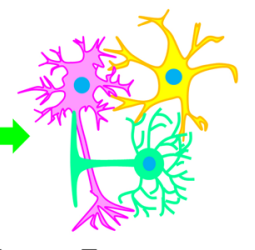

Treatment

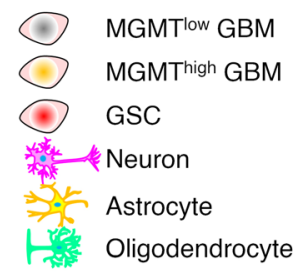

Figure 11. CK1A and BTRCP/MBD3 signaling regulates enrichment of MBD3-NuRD transcription repressive complex components and acetyl-histone H3 activity on neural differentiation-associated gene promoters. (A-D) ChIP-qPCR analysis of MBD3, HDAC1, HDAC2, MTA1, and acetyl-histone H3 occupancy at the MBD3-binding locus in U118MG or U251MG cells treated with DMSO $(n=3)$ and Pyr-Pam $(n=3)$. Immunoglobulin G (IgG) ChIP served as a negative control. Values are normalized to input control and represent the mean $\pm \mathrm{SD}$. ${ }^{*} P<0.05 ;{ }^{*} P<0.005 ;{ }^{* * *} P<0.0005$ by unpaired, 2-tailed Student's $t$ test. (E) Graphical summary showing that activation of CK1A signaling by Pyr-Pam induces proteasomal degradation of MBD3 and neural differentiation of GSCs through inhibiting enrichment of the MBD3-NuRD complex on neurogenesis-associated gene loci, leading to decreased GBM recurrence and growth. NuRD, nucleosome-remodeling and deacetylation.

bones. A burr hole was made through the skull at $1 \mathrm{~mm}$ posterior to bregma and $3 \mathrm{~mm}$ right of midline without breaking the dura. A 1/32 inch electric drill needle was inserted $5 \mathrm{~mm}$ ventral to the dura and retracted $0.5 \mathrm{~mm}$ (for injection), and then various sorted or unsorted cells were implanted stereotaxically at an infusion rate of $1 \mu \mathrm{L} / \mathrm{min}$ using a microcapillary tube needle. A total of $5 \times 10^{5}$ cells per mouse was administered. The needle was held in place 2-3 minutes to allow pressure equilibration within the cranial vault and then removed slowly and the hole immediately sealed with sterile bone wax to prevent leakage. Animals recovered from anesthesia and were returned to the animal care facility. For drug administration, micro-osmotic pumps (model 1004D, Alzet) and brain infusion kits were used to deliver drugs or vehicle into regions of the brain parenchyma or into the lateral ventricle via implanted cannulas, intrathecally (i.t.). Osmotic pumps were incubated 1 hour in sterile saline at $37^{\circ} \mathrm{C}$ before implantation and then filled with vehicle $(100 \mu \mathrm{L})$ or Pyr-Pam $(100 \mu \mathrm{L}$ at $7 \mathrm{mg} / \mathrm{mL})$ or TMZ $(100 \mu \mathrm{L}$ at $32.5 \mathrm{mg} / \mathrm{mL})$ in vehicle solution filled with saline. For PDX studies using recurrent human GBM tumors, we used recurrent human patient-derived tumors after both TMZ (11 cycles, 200-350 mg per administration) and radiation (dose: 60 Gy in 30 fractions) treatments. For implantation, tumor cells were resuspended in $1 \mathrm{~mL}$ Matrigel and implanted into brains using a Hamilton syringe (coordinates: $2 \mathrm{~mm}$ anterior and $3 \mathrm{~mm}$ to the right of bregma at a depth of 3 $\mathrm{mm})$. After injection, the surface was cleaned and the brain infusion 
cannula (Brain Infusion Kit 3, 1-3 mm; Alzet) was placed in the injection site and fixed to the skull using cyanoacrylate adhesive (Alzet). Filled pumps were connected to the brain infusion cannula according to the manufacturer's instructions and implanted s.c., delivering 20-25 $\mu$ g Pyr-Pam or $125 \mu$ T TMZ per day or vehicle only for 26 days. Tumor growth was monitored using an IVIS Spectrum imaging system (PerkinElmer) 2 times per week. In xenografts of patient-derived recurrent GBM tumors, cells dissociated from tumors in $200 \mu \mathrm{L}$ PBS/ Matrigel (1:1) were subcutaneously injected into the dorsal flank of immunocompromised (NSG) mice ( $n=4$ or 5 mice per group). Tumor volumes were measured every 4 days using Vernier calipers, applying the formula $\pi / 6 \times$ length $\times$ width $\times$ height. Immunohistochemistry was performed on paraffin-embedded tissue. For tissue preparation, tumors from xenograft mice were fixed and then paraffin-embedded immediately after mice were euthanized.

Cell culture. NPCs were prepared from the cortex of E11.5 mouse embryos in Hanks' balanced salt solution (HBSS, Invitrogen) and cultured as described previously (13). To maintain stem cell characteristics, adherent NPCs or GSCs were cultured in N2/B27 (1:1) medium containing bFGF. LN229 (CRL-2611), U87MG (HTB-14), U118MG (HTB-15), and T98G (CRL-1690) were purchased from ATCC and U251MG (09063001) was purchased from MilliporeSigma. The primary GSC line (USC02) was a gift from Tomas C. Chen, Florence M. Hofman, and Hee-Yeon Cho from the Neurological Surgery Department of the Keck School of Medicine at the University of Southern California (51). Cells were stored in CRYO-GOLD medium (Revive Organtech Inc). All cell lines were routinely tested for mycoplasma during experimental use. Stemness of cultured cells was confirmed by nestin and Pax6 expression. Spheres were established from suspended NPCs, astrocytes, or GBM cells and were maintained in N2/B27 (1:1) medium with bFGF (10 ng/mL) and epidermal growth factor (EGF; $20 \mathrm{ng} / \mathrm{mL}$ ) in ultra-low-attachment plates. To induce NPC or GSC differentiation, adherent cells were seeded and cultured in the absence of bFGF, and spheres were cultured in the absence of bFGF and EGF. To generate TMZ-resistant LN229, U118MG, U87MG, T98G, and U251MG lines, we employed methods previously described (51). Briefly, cell lines were incubated with doses of TMZ ranging from 10 to $100 \mathrm{mmol} / \mathrm{L}$ for approximately 3 months and then treated with 100 $\mathrm{mmol} / \mathrm{L}$ TMZ every other week. This method was designed to parallel development of TMZ resistance in the clinic, where GBMs initially sensitive to TMZ become subsequently resistant.

Bioluminescence imaging. Imaging of Fluc-expressing 9LLUCU118MG-MGMT ${ }^{\mathrm{lo}}$, -U251MG-MGMThi, -T98G, and -T98G GSCs was performed using an IVIS Spectrum imaging system. For in vivo imaging, animals were injected intraperitoneally with $150 \mathrm{mg} / \mathrm{kg}$ D-luciferin (Cayman Chemical) 5 minutes before imaging, at indicated times after implantation. Imaging times were 1-2 minutes per mouse, and photons emitted from specific regions were quantified over a defined time period ranging up to 3 minutes using Living Image software (Xenogen Corp.). In vivo luciferase activity is reported as photons per second.

Quantitative RT-PCR. Cells were harvested and total RNA isolated using TRIzol reagent (Invitrogen). The SuperScript III qRT-PCR kit (Invitrogen) was used to synthesize cDNA from total RNA. Quantitative PCR was carried out using the ABI PRISM 7900 Sequence Detection System with SYBR Green Master Mix (iTaq) with conditions of $95^{\circ} \mathrm{C}$ for 10 minutes followed by 50 cycles at $95^{\circ} \mathrm{C}$ for 15 seconds and $60^{\circ} \mathrm{C}$ for 3 seconds. Samples were run in triplicate and $M B D 3, C D 44$,
CD133, CXCR4, OCT4, SOX2, NANOG, KLF4, NESTIN, PAX6, BMI1, TUJ1, DLX1, NeuroD1, GAD67, GFAP, S10OB, MBP, and MAG transcript quantification was undertaken by comparing cycle threshold (Ct) values for each reaction with $G A P D H$ as the reference. Primers used in this study are listed in Supplemental Table 7.

Antibodies and reagents. Antibodies used in this study were antiCD44, anti-CK1A, and anti-MGMT (Novus Biologicals); anti-CXCR4 and anti-CD133 (5E3 clone) (Thermo Fisher Scientific); anti-HA (rabbit), anti-MBD3, and anti-BTRCP (Cell Signaling Technology); anti-Flag and anti-Ki67 (MilliporeSigma); anti-GST, anti- $\alpha$-tubulin, anti-MBD2, and anti-HA (mouse) (Santa Cruz Biotechnology); antiIgG, anti-histone H3 (acetyl-K27), anti-histone H3 (acetyl-K9 + K14 + $\mathrm{K} 18+\mathrm{K} 23$ + K27), anti-nestin, and anti-phosphoserine (Abcam); antiTuj1 (Covance); anti-Myc and anti-GFAP (Cell Signaling Technology); CD133/1 (AC133)-PE, human (clone AC133); and CD44-APC, human (clone DB105) (Miltenyi Biotec); and CXCR4-PEcy7 (BioLegend). Secondary antibodies were anti-rabbit Alexa Fluor 488-, anti-mouse Alexa Fluor 488-, anti-rabbit Alexa Fluor 555-, or anti-mouse Alexa Fluor 555-conjugated IgG (Thermo Fisher Scientific). bFGF and EGF were purchased from PeproTech. Protease inhibitor cocktail was from Roche Applied Science. TMX, Pyr-Pam, Protein A/G agarose beads, and 4'-6-diamidino-2-phenylindole (DAPI) were from MillporeSigma. D4476 was from Selleck. The ECL Kit and KOD Hot Start DNA polymerase were from MilliporeSigma. Glutathione magnetic beads, phenol/chloroform/isoamyl alcohol, and the First-Strand cDNA Synthesis Kit were from Thermo Fisher Scientific. Details of antibodies used in this study are listed in Supplemental Table 8.

IP, in vivo ubiquitination assay, and immunoblotting. IP and in vivo ubiquitination assays were performed as described previously (13). Briefly, cells were lysed with IP buffer (50 mM Tris-HCl, $\mathrm{pH}$ 7.4, $130 \mathrm{mM} \mathrm{NaCl}, 10 \mathrm{mM} \mathrm{NaF}, 2 \mathrm{mM}$ EGTA, 2 mM EDTA, 0.5\% Triton $\mathrm{X}-100,0.5 \% \mathrm{NP}-40,5 \%$ glycerol, $1 \mathrm{mM}$ dithiothreitol, and a protease inhibitor cocktail) for 1 hour on ice and centrifuged at $25,000 \mathrm{~g}$ at $4^{\circ} \mathrm{C}$ for 15 minutes. Supernatants were collected and precleared with 30 $\mu \mathrm{L}$ of Protein A/G beads (Santa Cruz Biotechnology) for 2 hours and then incubated with $4 \mu \mathrm{g}$ each of specific antibodies overnight at $4^{\circ} \mathrm{C}$. Lysates were then incubated with $30 \mu \mathrm{L}$ of Protein $\mathrm{A} / \mathrm{G}$ beads for 4 hours at $4^{\circ} \mathrm{C}$. After immune complexes were washed 6 times with IP buffer, they were eluted by boiling for 3 minutes at $95^{\circ} \mathrm{C}$ in SDS sample buffer and immunoblotted, as described previously(13).

Immunohistochemistry and immunocytochemistry. For immunohistochemistry, human primary GBM patient tissues, xenograft tumors, TMA samples, and embryonic brain tissues were dissected and fixed in $4 \%$ paraformaldehyde (PFA) in PBS at $4^{\circ} \mathrm{C}$ for 48 hours and then embedded in paraffin. For immunocytochemistry, cells cultured on coverslips were fixed with $4 \%$ PFA/PBS overnight and immunostained after permeabilizing with $0.2 \%$ Triton X-100. Tissues and cells were incubated with secondary antibodies at room temperature for 1 hour and counterstained in DAPI. Images were obtained using confocal microscopy (LSM5 PASCAL, Zeiss). Values obtained from at least 3 independent experiments were averaged and reported as means \pm SD.

Plasmids and shRNA transfection. The pcDNA3.0-HA-Ub and pcDNA3.0-CK1A-HA plasmids were a gift from KY Choi (Yonsei University, Seoul, South Korea). pcDNA3.1-Flag-BTRCP plasmids (full-length and $\triangle \mathrm{N}$ and $\triangle \mathrm{C}$ mutant forms) were provided by Jae Jung (University of Southern California). To knock down MBD3 or CK1A in human GBM cells, we generated a pLKO.1 shRNA lentiviral vector system. For 
both, sequences targeting $M B D 3$ or $C K 1 A$ were chosen using the RNAi Consortium shRNA library (http://www.broadinstitute.org/rnai/ public/) (3 clones targeting different sequences in 3 coding or 5'UTR regions of each gene). To generate oligonucleotides for cloning, sense and antisense sequences of chosen target sequences were ordered from IDT. Sequence-verified shRNA lentiviral plasmid vectors for human $M B D 3$ and $C K 1 A$ genes were subcloned into the pLKO.1 vector, and BTRCP siRNA was purchased from Santa Cruz Biotechnology. $M B D 3$ knockdown efficiencies were determined by Western blotting (see Supplemental Figure 5A).

MS. T98G GBM cells stably expressing Flag-MBD3 were collected and lysed with Tris lysis buffer. After centrifugation, supernatants were precleared with Protein A/G beads at $4^{\circ} \mathrm{C}$ for 2 hours and mixed with mouse anti-Flag antibody and protein $\mathrm{A} / \mathrm{G}$ beads for 4 hours at $4^{\circ} \mathrm{C}$. Precipitates were washed extensively with lysis buffer and resolved by SDS-PAGE. After silver staining, specific protein bands were excised and analyzed by ion-trap MS at the Harvard Taplin Biological Mass Spectrometry facility (https://taplin.med.harvard.edu/ home). Amino acid sequences were determined by tandem MS and database searches.

ChIP-qPCR and ChIP-seq. For the ChIP assay, GBM cell lines (LN229, U118MG, U87MG, U251MG, and T98G) or patient-derived primary GBM cells infected with $4 \mu \mathrm{g}$ pLKO.1 shMBD3 or a LKO.1 shScramble lentiviral vector for MBD3 loss-of-function experiments and/or treated with DMSO or $1 \mu \mathrm{g} / \mathrm{mL}$ Pyr-Pam for CK1A activation were treated with $1 \%$ formaldehyde for 10 minutes at room temperature and quenched with $0.125 \mathrm{M}$ glycine for 10 more minutes at room temperature. Cross-linked chromatin was sonicated to fragment DNA to 200-1,000 bp, and then immunoprecipitation was performed with rabbit anti-IgG and anti-acetyl-histone H3 (both Abcam); antiMBD3, anti-HDAC1, anti-HDAC2, and anti-MTA1 (all Cell Signaling Technology) antibodies overnight at $4^{\circ} \mathrm{C}$, followed by incubation with $50 \mu \mathrm{L}$ of magnetic Protein A/G Dynabeads (MilliporeSigma). Abundance of sequences in immunoprecipitates was determined by PCR and normalized as the fold-change relative to input chromatin. For analysis of MBD3 ChIP-seq data, we used the Integrative Genomics
Viewer (IGV v2.3) to visualize distribution of ChIP-seq-identified peaks in different genomic regions. Primer sets for ChIP-qPCR are listed in Supplemental Table 6.

Statitstics. Statistical analyses were performed using Excel statistical tools or Prism 5 (GraphPad Software). Statistical differences among 2 groups were analyzed using Student's $t$ test. One-way ANOVA tests (Tukey's multiple-comparison test) and 2-way ANOVA tests (Bonferroni's post hoc test) were used to test effects in multiple groups. A $P$ value less than 0.05 was considered significant: ${ }^{*} P<0.05$, ${ }^{* *} P<0.005,{ }^{* * *} P<0.0005$.

Study approval. Human specimens were approved by the IRB of the University of Southern California and all animal procedures were approved by the Institutional Animal Care and Use Committee (IACUC) of the University of Southern California.

\section{Author contributions}

BSM performed most experiments, analyzed data, designed the study, and wrote the manuscript. MC, TZ, and XS performed bulk sequencing analysis and helped with interpret RNA sequencing results. MY and GL helped with the PDX model and in vivo imaging. FJA and SLG provided patient samples. BSM and WL conceived of and supervised the study.

\section{Acknowledgments}

We thank members of Thomas C. Chen's laboratory for insightful discussions and Axel H. Schönthal (Molecular Microbiology \& Immunology, Keck School of Medicine, USC) for technical advice. BSM was supported by a fellowship from the CIRM (no. TG201161) and KRICT (SI2031-50).

Address correspondence to: Byoung-San Moon, Chonnam National University, Yeosu, 59626, Korea. Phone: 82.10.4017.7806; Email: bsmoon@jnu.ac.kr. Or to: Wange Lu, State Key Laboratory of Medicinal Chemical Biology and College of Life Sciences, Nankai University, 94 Weijin Road, 300071 Tianjin, China. Phone: 626.354.0759; Email: wangelv@gmail.com.
1. Legler JM, et al. Cancer surveillance series [corrected]: brain and other central nervous system cancers: recent trends in incidence and mortality. J Natl Cancer Inst. 1999;91(16):1382-1390.

2. Singh SK, et al. Identification of human brain tumour initiating cells. Nature. 2004;432(7015):396-401.

3. Bao S, et al. Glioma stem cells promote radioresistance by preferential activation of the DNA damage response. Nature. 2006;444(7120):756-760.

4. Beck B, Blanpain C. Unravelling cancer stem cell potential. Nat Rev Cancer. 2013;13(10):727-738.

5. Sanai N, Alvarez-Buylla A, Berger MS. Neural stem cells and the origin of gliomas. NEngl JMed. 2005;353(8):811-822.

6. Galli R, et al. Isolation and characterization of tumorigenic, stem-like neural precursors from human glioblastoma. Cancer Res. 2004;64(19):7011-7021.

7. Lee JH, et al. Human glioblastoma arises from subventricular zone cells with low-level driver mutations. Nature. 2018;560(7717):243-247.
8. Yao B, Christian KM, He C, Jin P, Ming GL, Song H. Epigenetic mechanisms in neurogenesis. Nat Rev Neurosci. 2016;17(9):537-549.

9. Wen S, Li H, Liu J. Dynamic signaling for neural stem cell fate determination. Cell Adh Migr. 2009;3(1):107-117.

10. Swartling FJ, Bolin S, Phillips JJ, Persson AI. Signals that regulate the oncogenic fate of neural stem cells and progenitors. Exp Neurol. 2014;260:56-68.

11. Mack SC, Hubert CG, Miller TE, Taylor MD, Rich JN. An epigenetic gateway to brain tumor cell identity. Nat Neurosci. 2016;19(1):10-19.

12. Noh EJ, Lim DS, Lee JS. A novel role for methyl CpG-binding domain protein 3, a component of the histone deacetylase complex, in regulation of cell cycle progression and cell death. Biochem Biophys Res Commun. 2009;378(3):332-337.

13. Moon BS, et al. Smek promotes corticogenesis through regulating Mbd3's stability and $\mathrm{Mbd} 3 / \mathrm{NuRD}$ complex recruitment to genes associated with neurogenesis. PLoS Biol. 2017;15(5):e2001220.
14. Kaji K, Caballero IM, MacLeod R, Nichols J, Wilson VA, Hendrich B. The NuRD component $\mathrm{Mbd} 3$ is required for pluripotency of embryonic stem cells. Nat Cell Biol. 2006;8(3):285-292.

15. Hendrich B, Bird A. Identification and characterization of a family of mammalian methyl-CpG binding proteins. Mol Cell Biol. 1998;18(11):6538-6547.

16. Hendrich B, Guy J, Ramsahoye B, Wilson VA, Bird A. Closely related proteins $\mathrm{MBD} 2$ and $\mathrm{MBD} 3$ play distinctive but interacting roles in mouse development. Genes Dev. 2001;15(6):710-723.

17. Cross SH, Meehan RR, Nan X, Bird A. A component of the transcriptional repressor MeCP1 shares a motif with DNA methyltransferase and HRX proteins. Nat Genet. 1997;16(3):256-259.

18. Lewis JD, et al. Purification, sequence, and cellular localization of a novel chromosomal protein that binds to methylated DNA. Cell. 1992;69(6):905-914.

19. Yildirim O, et al. Mbd3/NURD complex regulates expression of 5-hydroxymethylcytosine marked genes in embryonic stem cells. Cell. 
2011;147(7):1498-1510.

20. Zhang Y, Ng HH, Erdjument-Bromage H, Tempst P, Bird A, Reinberg D. Analysis of the NuRD subunits reveals a histone deacetylase core complex and a connection with DNA methylation. Genes Dev. 1999;13(15):1924-1935.

21. Rais Y, et al. Deterministic direct reprogramming of somatic cells to pluripotency. Nature. 2013;502(7469):65-70.

22. dos Santos RL, et al. MBD3/NuRD facilitates induction of pluripotency in a context-dependent manner. Cell Stem Cell. 2014;15(1):102-110.

23. Reynolds N, et al. NuRD suppresses pluripotency gene expression to promote transcriptional heterogeneity and lineage commitment. Cell Stem Cell. 2012;10(5):583-594.

24. Wade PA, Gegonne A, Jones PL, Ballestar E, Aubry F, Wolffe AP. Mi-2 complex couples DNA methylation to chromatin remodelling and histone deacetylation. Nat Genet. 1999;23(1):62-66.

25. Aguilera C, Nakagawa K, Sancho R, Chakraborty A, Hendrich B, Behrens A. c-Jun N-terminal phosphorylation antagonises recruitment of the Mbd3/NuRD repressor complex. Nature. 2011;469(7329):231-235.

26. Le Guezennec X, et al. MBD2/NuRD and MBD3/ $\mathrm{NuRD}$, two distinct complexes with different biochemical and functional properties. Mol Cell Biol. 2006;26(3):843-851.

27. Li R, et al. MBD3 inhibits formation of liver cancer stem cells. Oncotarget. 2017;8(4):6067-6078.

28. Fuchs SY, Spiegelman VS, Kumar KG. The many faces of beta-TrCP E3 ubiquitin ligases: reflections in the magic mirror of cancer. Oncogene. 2004;23(11):2028-2036.

29. Brescia P, Richichi C, Pelicci G. Current strategies for identification of glioma stem cells: adequate or unsatisfactory? J Oncol. 2012;2012:376894.

30. Beier D, et al. CD133(+) and CD133(-) glioblastoma- derived cancer stem cells show differential growth characteristics and molecular profiles. Cancer Res. 2007;67(9):4010-4015.

31. Clément V, Dutoit V, Marino D, Dietrich PY, Radovanovic I. Limits of CD133 as a marker of glioma self-renewing cells. Int J Cancer. 2009;125(1):244-248.

32. Song $X$, et al. Circular RNA profile in gliomas revealed by identification tool UROBORUS. Nucleic Acids Res. 2016;44(9):e87.

33. Phillips HS, et al. Molecular subclasses of highgrade glioma predict prognosis, delineate a pattern of disease progression, and resemble stages in neurogenesis. Cancer Cell. 2006;9(3):157-173.

34. Frescas D, Pagano M. Deregulated proteolysis by the F-box proteins SKP2 and beta-TrCP: tipping the scales of cancer. Nat Rev Cancer. 2008;8(6):438-449.

35. Marín-Ramos NI, et al. NEO212 inhibits migration and invasion of glioma stem cells. Mol Cancer Ther. 2018;17(3):625-637.

36. Kitange GJ, et al. Induction of MGMT expression is associated with temozolomide resistance in glioblastoma xenografts. Neuro-oncology. 2009;11(3):281-291.

37. Clement V, Sanchez P, de Tribolet N, Radovanovic I, Ruiz i Altaba A. HEDGEHOG-GLI1 signaling regulates human glioma growth, cancer stem cell self-renewal, and tumorigenicity. Curr Biol. 2007;17(2):165-172.

38. Wu L, et al. BCL3 expression promotes resistance to alkylating chemotherapy in gliomas. Sci Transl Med. 2018;10(448):eaar2238.

39. Yan H, et al. IDH1 and IDH2 mutations in gliomas. NEnglJMed. 2009;360(8):765-773.

40. Yang P, et al. IDH mutation and MGMT promoter methylation in glioblastoma: results of a prospective registry. Oncotarget. 2015;6(38):40896-40906.
41. Shimbo T, et al. MBD3 localizes at promoters, gene bodies and enhancers of active genes. PLOS Genet. 2013;9(12):e1004028.

42. Lathia JD, Mack SC, Mulkearns-Hubert EE, Valentim CL, Rich JN. Cancer stem cells in glioblastoma. Genes Dev. 2015;29(12):1203-1217.

43. Ehtesham M, Mapara KY, Stevenson CB, Thompson RC. CXCR4 mediates the proliferation of glioblastoma progenitor cells. Cancer Lett. 2009;274(2):305-312.

44. Anido J, et al. TGF- $\beta$ receptor inhibitors target the CD44(high)/Id1(high) glioma-initiating cell population in human glioblastoma. Cancer Cell. 2010;18(6):655-668.

45. Thorne CA, et al. Small-molecule inhibition of Wnt signaling through activation of casein kinase 1a. Nat Chem Biol. 2010;6(11):829-836.

46. Cui Y, et al. Regulatory landscape and clinical implication of MBD3 in human malignant glioma. Oncotarget. 2016;7(49):81698-81714.

47. Bady P, et al. DNA fingerprinting of glioma cell lines and considerations on similarity measurements. Neuro-oncology. 2012;14(6):701-711.

48. Ozawa T, Wang J, Hu LJ, Lamborn KR, Bollen AW, Deen DF. Characterization of human glioblastoma xenograft growth in athymic mice. In Vivo. 1998;12(4):369-374.

49. Lewis-Tuffin LJ, et al. Misregulated E-cadherin expression associated with an aggressive brain tumor phenotype. PLoS One. 2010;5(10):e13665

50. Bao ZS, et al. RNA-seq of 272 gliomas revealed a novel, recurrent PTPRZ1-MET fusion transcript in secondary glioblastomas. Genome Res. 2014;24(11):1765-1773.

51. Cho HY, et al. NEO212, temozolomide conjugated to perillyl alcohol, is a novel drug for effective treatment of a broad range of temozolomide-resistant gliomas. Mol Cancer Ther. 2014;13(8):2004-2017. 